$\underline{\text { Preprint typeset in JHEP style - HYPER VERSION }}$

SISSA $/ 45 / 2009 / \mathrm{EP}$

ULB-TH/09-25

hep-th/0908.0056

\title{
Ghost story. III. Back to ghost number zero
}

\author{
L.Bonora \\ International School for Advanced Studies (SISSA/ISAS) \\ Via Beirut 2-4, 34014 Trieste, Italy, and INFN, Sezione di Trieste \\ E-mail: bonora@sissa.it, \\ C.Maccaferri \\ International Solvay Institutes and Physique Théorique et Mathématique, \\ ULB C.P. 231, Université Libre de Bruxelles, B-1050, Bruxelles, Belgium \\ E-mail: cmaccafe@ulb.ac.be, \\ D.D.Tolla \\ Department of Physics and University College, Sungkyunkwan University, Suwon \\ 440-746, South Korea \\ E-mail: ddtolla@skku.edu
}

\begin{abstract}
After having defined a 3-strings midpoint-inserted vertex for the $b c$ system, we analyze the relation between $g h=0$ states (wedge states) and $g h=3$ midpoint duals. We find explicit and regular relations connecting the two objects. In the case of wedge states this allows us to write down a spectral decomposition for the $g h=0$ Neumann matrices, despite the fact that they are not commuting with the matrix representation of $K_{1}$. We thus trace back the origin of this noncommutativity to be a consequence of the imaginary poles of the wedge eigenvalues in the complex $\kappa$-plane. With explicit reconstruction formulas at hand for both $g h=0$ and $g h=3$, we can finally show how the midpoint vertex avoids this intrinsic noncommutativity at $g h=0$, making everything as simple as the zero momentum matter sector.
\end{abstract}

KeYwords: String Field Theory, Ghost Wedge States. 


\section{Contents}

1. Introduction

2. Overview 1

2.1 Wedge States

$2.2 Y( \pm i)$-inserted wedge states

2.2.1 Principal $g h=3$ wedges

3. Reconstruction formulas for $g h=3$ wedges 8

3.1 Principal $g h=3$ wedges 8

$3.2 Y( \pm i)$-inserted $g h=3$ wedges 9

4. The relation between $g h=\mathbf{3}$ and $g h=\mathbf{0}$

4.1 The midpoint reflector 11

4.2 Midpoint Identities 13

4.2.1 Midpoint identities for 'principal' states 14

4.2.2 Midpoint identities for $Y(i)$-inserted wedges

4.2.3 Midpoint identities for the vertex 16

$4.3 \quad g h=0$ from $g h=3$ : zero modes coefficients 17

4.3.1 A check: $g h=0$ zero mode coefficients of surface states 19

$4.4 \quad g h=0$ from $g h=3$ : bulk reconstruction 20

4.5 Normalization 24

5. Midpoint multiplication of $g h=0$ squeezed states

6. Conclusions and discussion 28

A. BRST invariant squeezed states 30

A.1 BRST invariant $g h=0 / 3$ wedges 32

B. Irrelevance of secondary poles 34

\section{Introduction}

This is the third and conclusive paper of a series including [1], referred to as I, and [2], referred to as II; see also [3]. The whole story started up with a very simple question. In Open String Field Theory, 四, wedge states are surface states $|N\rangle$ labeled by a real (possibly integer) number $N$, obeying the star algebra relation

$$
|N\rangle *|M\rangle=|N+M-1\rangle .
$$


Wedge states, originally defined and studied in [5, 6, 7], have been a fundamental ingredient for constructing and analyzing, following the breakthrough by Schnabl [8], analytic solutions for the tachyon vacuum [9, 10, 11, 12, 13, 14, 15, 16], solutions describing marginal deformations 17, 18, 19, 20, 21, 22, 23, 24, 25, 26] and related topics 227, 28, 29, 30, 31, 32]. See [33] for a recent review and a guide through other references.

While this simple commutative $*$-subalgebra has a very intuitive realization in terms of gluing vertical strips in the arctan-sliver frame (or, equivalently, of gluing sectors of cones), the oscillator realization of this multiplication rule has only been understood in detail in the (non-universal) matter sector, [34, 35] (corresponding to $D$-free bosons) and in the (universal) bosonized ghost system, [36]. Strangely enough, the original (universal) bc system which naturally emerges from the Polyakov path integral, while quite similar to the matter sector in the conventional normal ordering on $c_{1}|0\rangle$, [37, 38, 39, 40, 41], turns out to give a quite complicated oscillator formalism, when one normal-orders with respect to the $S L(2, R)$-vacuum. This is due to several conspiring accidents but the very reason is the presence of the three zero modes of the $c$ ghost. Indeed, a closely related system, the $h=(1,0) b c$ system, having just one $c$ zero mode, turns out to be almost identical to the zero momentum matter sector, 42, 43.

In this paper we deal with the co-existence of the $g h=0$ and the $g h=3$ sectors. These two sectors are naturally conjugate to each other by the $b p z$ inner product. Still, while the $g h=0$ sector is a $*$-subalgebra, the $g h=3$ is not because the ghost number is additive under the $*$-product. A related issue is that, in general, there is no unique prescription to pair $g h=0$ and $g h=3$ states in a one-to-one way. Any insertion of $3 c$ 's operators could in principle do the job. There are however better choices than others. In particular one would like to pair $g h=0$ and $g h=3$ states in such a way that they retain the same conformal properties. This implies the use of a $g h=3$ insertion with zero conformal weight. In the ghost sector, there is just one such field, given by

$$
Y(z)=\frac{1}{2} \partial^{2} c \partial c c(z) .
$$

This composite field, also considered in [44] in the context of VSFT [45], has a number of nice properties

- It is a weight zero primary

$$
T(z) Y(w)=\frac{\partial Y(w)}{z-w}+\ldots
$$

- It is BRST invariant

$$
\left\{Q_{B}, Y(z)\right\}=\oint_{z} \frac{d w}{2 \pi i} j_{B}(w) Y(z)=0
$$

- Its derivative is $Q_{B}$-exact

$$
\partial Y(z)=\left[Q_{B}, \partial^{2} c \partial c(z)\right]
$$


This implies that, given two BRST invariant $g h=0$ states (for example surface states) we have

$$
\partial_{z}\left\langle\psi_{1}|Y(z)| \psi_{2}\right\rangle=0
$$

So, for BRST-closed states, correlators are independent of the location of $Y$.

However OSFT deals with off shell states which need not be BRST-invariant. Moreover the $*$-product breaks conformal invariance down to the subgroup of midpoint preserving reparametrizations. This implies that, out of the infinite places where one could insert $Y$, the only one which is not going to interfere is the midpoint. In general, when dealing with conformal maps such as the ones which define the $*$-product, having midpoint insertions is forbidden because such maps are singular precisely at the midpoint. The only exception is when we are dealing with weight zero primaries, which will not pick up any singular Jacobian from the conformal transformation. Once again, at $g h=3$, this uniquely selects the operator $Y$. Concretely, the midpoint insertion of $Y$ commutes with the generators of midpoint preserving reparametrizations

$$
\left[K_{n}, Y( \pm i)\right]=0
$$

Here we will be mostly interested in $K_{1}$-invariance, since this symmetry allowed, in all previously known cases, [46, 36, 47] to diagonalize the Neumann matrices of the 3-string vertex.

Given the above reasons, for any state at $g h=0, \psi$, we define its midpoint dual(s) to be

$$
\hat{\psi}_{( \pm i)} \equiv Y( \pm i) \psi
$$

This relation is not a one to one map in general because of the non trivial kernel of $Y(i)$ but it is effectively so for $g h=0$ states which do not have $c$ insertions at the midpoint. All $g h=0$ squeezed states (and in particular surface states) are in this class.

In this paper we deal with $g h=0$ and $g h=3$ squeezed states, where the latter are intended to be obtained from the former by the insertion of $Y$ at one of the two midpoints $z= \pm i$. By using the method of images (doubling trick) one can associate two $g h=3$ states to each $g h=0$ state

$$
\hat{\psi} \equiv Y\left(\frac{\pi}{2}\right) \psi=\frac{1}{2}(Y(i)+Y(-i)) \psi=\frac{1}{2}\left(\hat{\psi}_{(i)} \oplus \hat{\psi}_{(-i)}\right) \simeq\left(\hat{\psi}_{(i)}, \hat{\psi}_{(-i)}\right) .
$$

The reason for the above notation is that, under the $*$-product, squeezed states are a ring rather than an algebra. This just means that we will separately consider states with a holomorphic and an anti-holomorphic midpoint insertion, because their half sum is not a squeezed state anymore.

One of the main problems we address in this paper is how to get back, in the case of squeezed states (and, in particular, of surface states), the $g h=0$ states by knowing their 
$g h=3$ cousins. To be more specific the $Y$ midpoint insertion will be implemented via a 2 -strings vertex (midpoint reflector) which is a squeezed state of total ghost number 6 . The ordinary 2 -strings vertex (implementing the $b p z$ conjugation) would have total ghost number 3. So the two differ by 3 units of ghost number, which are provided by the $Y$ insertion.

We will see that $g h=3$ squeezed states which are obtained by applying $Y( \pm i)$ to $g h=0$ squeezed states, are characterized by having a Neumann matrix whose zero mode part is uniquely determined by the non zero mode part. We call this property midpoint identity. The explicit knowledge of both $Y( \pm i)$-inserted $g h=3$ squeezed states, will allow us to reconstruct the original $g h=0$ squeezed state. We will provide evidence that this is possible at least for all (non-projectors) surface states.

After analyzing in detail the $g h=0 / g h=3$ relations in the case of wedge states (which are the only $K_{1}$ invariant surface states), we will finally $*$-multiply them via the midpoint 3 -string vertex defined in paper II. Here we will see how the midpoint identities of the vertex, together with the structure of the $g h=0$ Neumann matrices (which represent the states to be multiplied) conspire to effectively transform these $g h=0$ matrices into $g h=3$ ones. Differently from $g h=0$ case, $K_{1}$-invariance at $g h=3$ does imply commuting matrices, so the star product will be as easy as the zero momentum matter product. In particular all the matrices in the game will commute, so, trading them with their eigenvalues, they will reconstruct the star product of the two wedges with an overall $Y( \pm i)$ insertion. This insertion can then be undone by using once again the $g h=0 / g h=3$ relation and to finally get the $g h=0$ state which is just the star product of the $2 g h=0$ states. This completes the program started with I.

\section{Overview}

The aim of this section is to give an overview of the objects that will feature in this paper. We will deal with $g h=0$ states, the wedge states, and $g h=3$ states, wedge states with a midpoint $Y$ insertion. Both of them can be uniquely defined as squeezed states on the vacua $|0\rangle$ and $|\hat{0}\rangle \equiv c_{-1} c_{0} c_{1}|0\rangle$, via a $g h=0$ Neumann function and a $g h=3$ one, respectively. The two different Neumann functions are known once the conformal map is given. In the case of wedges, we fix $S L(2, C)$ invariance by choosing the conformal maps to be

$$
f_{N}(z)=\left(\frac{1+i z}{1-i z}\right)^{\frac{2}{N}}
$$

\subsection{Wedge States}

Wedge states are squeezed states on the $g h=0$ vacuum $|0\rangle$

$$
|N\rangle=e^{c_{P}^{\dagger} S_{P q} b_{q}^{\dagger}}|0\rangle
$$


with the (long-short) defining matrix given by

$$
\begin{aligned}
S_{M n}^{(N)}= & \oint_{0} \frac{d z}{2 \pi i} \oint_{0} \frac{d w}{2 \pi i} \frac{1}{z^{M-1}} \frac{1}{w^{n+2}} \\
& {\left[\frac{f_{N}^{\prime}(z)^{2}}{f_{N}^{\prime}(w)} \frac{1}{f_{N}(z)-f_{N}(w)}\left(\frac{f_{N}(w)-f_{N}(0)}{f_{N}(z)-f_{N}(0)}\right)^{3}-\frac{w^{3}}{z^{3}(z-w)}\right] . }
\end{aligned}
$$

( $M=-1,0,1, \ldots, n=2,3, \ldots$, see II for notation; moreover we will drop the label $N$ in $S^{(N)}$ whenever this is not strictly necessary). This matrix has the general bulk/zero mode block decomposition

$$
S=\left(\begin{array}{ll}
0 & s \\
0 & S
\end{array}\right),
$$

where the upper left 0 represents a vanishing $3 \times 3$ matrix, the lower left 0 represents three infinite short columns, $s$ represents three infinite short rows, while $S$ is the bulk. Wedge states are annihilated by $K_{1}=L_{1}+L_{-1}$. This can be checked explicitly by writing $K_{1}$ as

$$
K_{1}=c_{N}^{\dagger} G_{N M} b_{M}+b_{n}^{\dagger} H_{n m} c_{m}-3 c_{2} b_{-1}
$$

We get $K_{1}|N\rangle=0$ iff

$$
\left(G S+S H^{T}\right)_{N m}+3 S_{N 2} S_{-1 m}=0
$$

This relation can be explicitly checked by picking up residues in eq.(2.3).

Note that, despite the fact that $K_{1}|N\rangle=0$, the Neumann coefficients do not anti-commute with $G$ and $H$. Moreover the violation of anti-commutativity depends explicitly on the zero mode contribution of the $g h=0$ Neumann function. This is one of the main reasons why it will be necessary to insert the operator $Y$ at the midpoint and to consider $g h=3$ squeezed states, whose Neumann matrices will commute with $G$. We will see later on that the knowledge of $g h=3$ midpoint inserted squeezed states will imply the knowledge of the $g h=0$ ones, whose $Y( \pm i)$ inserted versions give the former.

\section{$2.2 Y( \pm i)$-inserted wedge states}

We can define wedge states (and surface states in general) with local operator insertions. In particular we are interested in the midpoint insertion of the BRST invariant, primary scalar $Y(z)=\frac{1}{2} \partial^{2} c \partial c c(z)$.

Let us begin by observing that a surface state (which is identified via an analytic map, $f(z)$, from the unit semidisk of the UHP, to a Riemann surface $\Sigma$ with the disk topology) with an insertion of $Y$ at a point $\xi$ on the Riemann surface can be written as a squeezed state on the $g h=3$ vacuum $\langle\hat{0}|=\langle 0| c_{-1} c_{0} c_{1}$ as ${ }^{1}$

$$
\left\langle\hat{S}^{\xi}\right|=\langle\hat{0}| e^{-c_{n} \hat{S}_{n M}^{(\xi)} b_{M}}
$$

\footnotetext{
${ }^{1}$ Note that there is no normalization arising from the transformation of the insertion, this is unambiguous because $Y$ is a zero-weight primary.
} 
where the (short-long) Neumann coefficients are given by

$$
\hat{S}_{n M}^{(\xi)}=\oint_{0} \frac{d z}{2 \pi i} \oint_{0} \frac{d w}{2 \pi i} \frac{1}{z^{n-1}} \frac{1}{w^{M+2}}\left[\frac{f^{\prime}(z)^{2}}{f^{\prime}(w)} \frac{1}{f(z)-f(w)}\left(\frac{f(w)-\xi}{f(z)-\xi}\right)^{3}-\frac{1}{z-w}\right]
$$

A midpoint insertion (which is only acceptable for zero-weight primaries) is given by $\xi=f( \pm i)$.

Let us concentrate on wedge states $|N\rangle$ whose analytic map (up to $S L(2, C)$ ) is given by $f_{N}(z)=\left(\frac{1+i z}{1-i z}\right)^{\frac{2}{N}}$. We have respectively

$$
\begin{aligned}
\langle N| Y(i) & \equiv\left\langle\hat{N}_{(i)}\right|=\langle\hat{0}| e^{-c_{n} \hat{S}_{n M}^{\left(f_{N}(i)\right)} b_{M}} \\
\langle N| Y(-i) & \equiv\left\langle\hat{N}_{(-i)}\right|=\langle\hat{0}| e^{-c_{n} \hat{S}_{n M}^{\left(f_{N}(-i)\right)} b_{M}},
\end{aligned}
$$

In order to lighten a bit the notation we will rename

$$
\hat{S}_{( \pm i)} \equiv \hat{S}^{\left(f_{N}( \pm i)\right)}
$$

and write the Neumann coefficients as $\left(f_{N}(i)=0, f_{N}(-i)=\infty\right)$

$$
\begin{aligned}
\hat{S}_{(i) n M} & =\oint_{0} \frac{d z}{2 \pi i} \oint_{0} \frac{d w}{2 \pi i} \frac{1}{z^{n-1}} \frac{1}{w^{M+2}}\left[\frac{f_{N}^{\prime}(z)^{2}}{f_{N}^{\prime}(w)} \frac{1}{f_{N}(z)-f_{N}(w)}\left(\frac{f_{N}(w)}{f_{N}(z)}\right)^{3}-\frac{1}{z-w}\right] \\
\hat{S}_{(-i) n M} & =\oint_{0} \frac{d z}{2 \pi i} \oint_{0} \frac{d w}{2 \pi i} \frac{1}{z^{n-1}} \frac{1}{w^{M+2}}\left[\frac{f_{N}^{\prime}(z)^{2}}{f_{N}^{\prime}(w)} \frac{1}{f_{N}(z)-f_{N}(w)}-\frac{1}{z-w}\right]
\end{aligned}
$$

It is easy to prove the twist property

$$
\hat{S}_{(i) n M}=\left(\hat{S}_{(-i) n M}\right)^{*}=(-1)^{m+N} \hat{S}_{(-i) n M} .
$$

From the fact that

$$
\left[K_{1}, Y( \pm i)\right]=0
$$

it follows that $Y( \pm i)$-inserted wedge states are also annihilated by $K_{1}=L_{1}+L_{-1}$. This can be checked explicitly by writing $K_{1}$ as

$$
K_{1}=c_{n}^{\dagger} H_{n m}^{T} b_{m}+b_{N}^{\dagger} G_{N M}^{T} c_{M}+3 c_{2}^{\dagger} b_{1}^{\dagger} .
$$

We get $\left\langle\hat{N}_{( \pm i)}\right| K_{1}=0$ iff

$$
\left(H^{T} \hat{S}_{( \pm i)}+\hat{S}_{( \pm i)} G\right)_{n M}+3 \delta_{n 2} \delta_{-1 M}=0
$$

and it is easy to check this directly (see II).

We see that the Neumann coefficients still do not (anti-)commute with $G$ and $H$. The violation is however very mild and, contrary to the $g h=0$ case, it is universal (it doesn't depend on the particular wedge state). 
As argued in II, these $g h=3$ matrices can be 'augmented' to auxiliary big matrices by adding the 'radial ordering' matrix

$$
z_{i j}=\delta_{i+j}, \quad i, j=-1,0,1
$$

to the $3 \times 3$ zero mode block, in the following way

$$
\hat{\mathbf{S}}_{( \pm i)}=\left(\begin{array}{cc}
z & 0 \\
\hat{s}_{( \pm i)} & \hat{S}_{( \pm i)}
\end{array}\right) .
$$

This extra term will not contribute to the state, because of normal ordering, and it is thus a freedom we can take. It is then immediate to check that

$$
G \hat{\mathbf{S}}_{( \pm i)}+\hat{\mathbf{S}}_{( \pm i)} G=0
$$

\subsubsection{Principal $g h=3$ wedges}

In order to understand the spectral decomposition of the above defined Neumann matrices (both at $g h=0$ and at $g h=3$ ), it will be useful to define auxiliary twist invariant $g h=3$ squeezed states, which we dubbed in II 'principal' $g h=3$ wedges. Principal wedge states are twist invariant squeezed states on the $g h=3$ vacuum $\langle\hat{0}| \equiv\langle 0| c_{-1} c_{0} c_{1}$

$$
\langle\hat{N}|=\langle\hat{0}| e^{-c_{n} \hat{S}_{n M} b_{M}},
$$

with the (short-long) defining matrix given $b y^{2}$

$$
\hat{S}_{p Q}=\oint_{0} \frac{d z}{2 \pi i} \oint_{0} \frac{d w}{2 \pi i} \frac{1}{z^{p-1}} \frac{1}{w^{Q+2}}\left(\frac{2 i}{N} \frac{1+w^{2}}{\left(1+z^{2}\right)^{2}} \frac{f_{N}(z)+f_{N}(w)}{f_{N}(z)-f_{N}(w)}-\frac{1}{z-w}\right)
$$

This matrix has the general form

$$
\hat{S}=\left(\begin{array}{ll}
0 & 0 \\
\hat{S} & \hat{S}
\end{array}\right) .
$$

We will see in the next section that these states differ from wedges with $Y( \pm i)$ insertion by a rank 2 matrix. It follows that they are not surface states with insertions and, consequently, they are not BRST invariant (see appendix A for a discussion concerning BRST invariance and the overlap between $g h=0$ and $g h=3$ squeezed states).

Their Neumann matrix is nonetheless the 'principal' part of all the $g h=0$ and $g h=3$ BRST invariant Neumann matrices. All other matrices will differ from the former by a rank 2 correction. Principal $g h=3$ wedge states are also annihilated by $K_{1}=L_{1}+L_{-1}$ and, as we saw in paper II, their bulk is completely reconstructed from the continuous spectrum, with the standard choice of contour given by the real axis of the complex $\kappa$-plane.

\footnotetext{
${ }^{2}$ This expression is not $S L(2, C)$ invariant. This is the first indication that these states are not surface states with insertions.
} 


\section{Reconstruction formulas for $g h=3$ wedges}

Since the $g h=3$ Neumann matrices (anti)-commute with $G$, the standard continuous plus discrete basis will reconstruct them starting from their eigenvalues. For the sake of clarity, here we briefly review some of the results derived in paper II.

\subsection{Principal $g h=3$ wedges}

These are the simplest states, as their bulk is just given by the real continuous spectrum, (and it is the 'principal part' for all the $g h=3 / 0$ states we will consider)

$$
\hat{S}_{p q}=\int d \kappa \frac{1}{2 \sinh \frac{\pi \kappa}{2}} \frac{\sinh \frac{\pi \kappa(2-N)}{4}}{\sinh \frac{\pi \kappa N}{4}} V_{p}^{(2)}(-\kappa) V_{q}^{(-1)}(\kappa), \quad p, q \geq 2 .
$$

The complete reconstruction, including the zero modes 3 -column and the radial ordering block $z_{i j}=\delta_{i+j}$, is given by the continuous + discrete spectrum

$$
\begin{aligned}
\hat{S}_{P Q} & =\int d \kappa \frac{1}{2 \sinh \frac{\pi \kappa}{2}} \frac{\sinh \frac{\pi \kappa(2-N)}{4}}{\sinh \frac{\pi \kappa N}{4}} V_{p}^{(2)}(-\kappa) V_{Q}^{(-1)}(\kappa) \\
& +\sum_{\xi= \pm 2 i, 0}(-1)^{\frac{\xi}{2 i}} \tilde{v}_{P}^{(2)}(-\xi) \tilde{V}_{Q}^{(-1)}(\xi) \\
& =\left(\begin{array}{cc}
z_{i j} & 0 \\
\hat{s}_{p i} & \hat{S}_{p q}
\end{array}\right) .
\end{aligned}
$$

We recall, from paper II, that the $\tilde{v}^{(2)}(\xi)$ discrete eigenvectors are given by

$$
\tilde{v}^{(2)}(0)=\frac{1}{\sqrt{2}}\left(\begin{array}{c}
1 \\
0 \\
1 \\
0 \\
-3 \\
0 \\
\vdots
\end{array}\right), \quad \tilde{v}^{(2)}( \pm 2 i)=\frac{1}{2}\left(\begin{array}{c}
1 \\
\mp i \\
-1 \\
\pm i \\
1 \\
\mp i \\
\vdots
\end{array}\right)
$$

while the $\tilde{V}_{Q}^{(-1)}(\xi)$ are just the $h=-1$ continuous eigenvectors, evaluated at $\xi=0, \pm 2 i$, with the same normalization as the $\tilde{v}^{(2)}(\xi)$ 's.

$$
\begin{aligned}
\tilde{V}^{(-1)}(0) & =\frac{1}{\sqrt{2}} V^{(-1)}(0)=\frac{1}{\sqrt{2}}(1,0,1 ; 0,0,0, \ldots) \\
\tilde{V}^{(-1)}( \pm 2 i) & =\frac{1}{2} V^{(-1)}( \pm 2 i)=\frac{1}{2}(1, \pm 2 i,-1 ; 0,0,0, \ldots) .
\end{aligned}
$$

These vectors just contain zero mode contributions. Moreover the continuous spectral measure for the identity operator, $\frac{1}{2 \sinh \frac{\pi \kappa}{2}}$, has simple poles precisely at $(\kappa=0, \pm 2 i)$. This can be viewed as the origin of the discrete spectrum (for a thorough discussion see II). 


\section{2 $Y( \pm i)$-inserted $g h=3$ wedges}

The Neumann functions of the $g h=3$ wedges $\left\langle\hat{N}_{( \pm i)}\right| \equiv\langle N| Y( \pm i)$, and the one of the 'principal' wedges $\langle\hat{N}|$, are very simply related. It is a matter of trivial algebra to check that for $\left\langle\hat{N}_{(i)}\right|$ we have

$$
\begin{aligned}
\hat{S}_{(i)}(z, w) & =\frac{f_{N}^{\prime}(z)^{2}}{f_{N}^{\prime}(w)} \frac{1}{f_{N}(z)-f_{N}(w)}\left(\frac{f_{N}(w)}{f_{N}(z)}\right)^{3} \\
& =\frac{2 i}{N} \frac{1+w^{2}}{\left(1+z^{2}\right)^{2}} \frac{f_{N}(z)+f_{N}(w)}{f_{N}(z)-f_{N}(w)}-\frac{4 i}{N} \frac{1+w^{2}}{\left(1+z^{2}\right)^{2}}\left(\frac{f_{N}(w)}{f_{N}(z)}+\frac{1}{2}\right),
\end{aligned}
$$

while for $\left\langle\hat{N}_{(-i)}\right|$

$$
\begin{aligned}
\hat{S}_{(-i)}(z, w) & =\frac{f_{N}^{\prime}(z)^{2}}{f_{N}^{\prime}(w)} \frac{1}{f_{N}(z)-f_{N}(w)} \\
& =\frac{2 i}{N} \frac{1+w^{2}}{\left(1+z^{2}\right)^{2}} \frac{f_{N}(z)+f_{N}(w)}{f_{N}(z)-f_{N}(w)}+\frac{4 i}{N} \frac{1+w^{2}}{\left(1+z^{2}\right)^{2}}\left(\frac{f_{N}(z)}{f_{N}(w)}+\frac{1}{2}\right) .
\end{aligned}
$$

This readily gives the following reconstruction formulas

$$
\begin{gathered}
\hat{S}_{(i)}(z, w)=\hat{S}(z, w)-\frac{4 i}{N}\left(f_{\kappa=-\frac{4 i}{N}}^{(2)}(z) f_{\kappa=\frac{4 i}{N}}^{(-1)}(w)+\frac{1}{2} f_{\kappa=0}^{(2)}(z) f_{\kappa=0}^{(-1)}(w)\right) \\
\hat{S}_{(-i)}(z, w)=\hat{S}(z, w)+\frac{4 i}{N}\left(f_{\kappa=\frac{4 i}{N}}^{(2)}(z) f_{\kappa=-\frac{4 i}{N}}^{(-1)}(w)+\frac{1}{2} f_{\kappa=0}^{(2)}(z) f_{\kappa=0}^{(-1)}(w)\right)
\end{gathered}
$$

where $\hat{S}(z, w)$ is the Neumann function for the 'principal' $g h=3$ wedges (whose bulk is just given by the continuous spectrum) and

$$
\begin{aligned}
f_{\kappa}^{(2)}(z) & =\frac{1}{\left(1+z^{2}\right)^{2}} e^{\kappa \tan ^{-1} z}=\sum_{n=2}^{\infty} V_{n}^{(2)}(\kappa) z^{-n+1} \\
f_{\kappa}^{(-1)}(z) & =\left(1+z^{2}\right) e^{\kappa \tan ^{-1} z}=\sum_{M=-1}^{\infty} V_{M}^{(-1)}(\kappa) z^{-M-2} .
\end{aligned}
$$

It is easy to see that the above reconstruction formulas can (almost) be obtained by integrating (we focus on $\hat{S}_{(i)}(z, w)$ ) on a path along the band $\frac{4}{N}<\Im(\kappa)<2$. This gives (in addition to the contribution along the real axis) the residue around $\kappa=\frac{4 i}{N}$ and half the residue at $\kappa=0$ (this pole counts $\frac{1}{2}$ because it sits on the real axis). Concretely

$$
\begin{aligned}
& \int_{\frac{4}{N}<\Im(\kappa)<2} d \kappa \frac{1}{2 \sinh \frac{\pi \kappa}{2}} \frac{\sinh \frac{\pi \kappa(2-N)}{4}}{\sinh \frac{\pi \kappa N}{4}} f_{-\kappa}^{(2)}(z) f_{\kappa}^{(-1)}(w)=\int_{R} d \kappa(\ldots)-\oint_{\frac{4 i}{N}} d \kappa(\ldots)-\frac{1}{2} \oint_{0} d \kappa(\ldots) \\
& =\left.\hat{S}(z, w)\right|_{\text {cont }}-\frac{4 i}{N} f_{\kappa=-\frac{4 i}{N}}^{(2)}(z) f_{\kappa=\frac{4 i}{N}}^{(-1)}(w)-\frac{i(2-N)}{N} f_{\kappa=0}^{(2)}(z) f_{\kappa=0}^{(-1)}(w) \\
& =\left.\hat{S}_{(i)}(z, w)\right|_{\text {cont }}+i f_{\kappa=0}^{(2)}(z) f_{\kappa=0}^{(-1)}(w),
\end{aligned}
$$

where by the subscript $\left.(\cdot)\right|_{\text {cont }}$, we indicate the contribution from just the continuous spectrum. 
One should notice that a small mismatch is present: the half residue around $\kappa=0$ gives an extra $i f_{\kappa=0}^{(2)}(z) f_{\kappa=0}^{(-1)}(w)$. It should be stressed however that this extra contribution is universal, i.e. it does not depend on the $N$ of the wedge state under consideration, moreover it only affects the 3-column of the Neumann matrix. We will see in a while that this extra universal contribution is not an unwelcome accident but a manifestation of a very important relation: the midpoint identity.

In conclusion, modulo universal stuff, $Y(i)$-inserted $g h=3$ wedges are precisely reconstructed by shifting the path of the $\kappa$ integration.

To this one should add the contribution from the discrete spectrum, which is the same as for 'principal' states. In total we thus have $\left(\xi_{N}=+\frac{4 i}{N}\right)$

$$
\begin{aligned}
\hat{S}_{(i) M Q} & =\int_{\Im(\kappa)>\Im\left(\xi_{N}\right)} d \kappa \frac{1}{2 \sinh \frac{\pi \kappa}{2}} \frac{\sinh \frac{\pi \kappa(2-N)}{4}}{\sinh \frac{\pi \kappa N}{4}} V_{m}^{(2)}(-\kappa) V_{Q}^{(-1)}(\kappa) \\
& +\sum_{\xi= \pm 2 i, 0}(-1)^{\frac{\xi}{2 i}} \tilde{v}_{M}^{(2)}(-\xi) \tilde{V}_{Q}^{(-1)}(\xi) \\
& -i P_{m Q} \\
& =\left(\begin{array}{cc}
z_{i j} & 0 \\
\hat{s}_{(i) p j} & \hat{S}_{(i) m q}
\end{array}\right),
\end{aligned}
$$

where, following $\mathrm{II}^{3}$, we have indicated

$$
P_{m Q}=V_{m}^{(2)}(0) V_{Q}^{(-1)}(0) .
$$

As anticipated above, the spurious $-i P_{m Q}$ contribution will find its rationale in the implementation of the midpoint identity, as we will see in the next section.

The same reconstruction obviously apply to $S_{(-i)}(z, w)$, by just integrating along the band $-2<\Im(\kappa)<-\frac{4}{N}$. Explicitly

$$
\begin{aligned}
\hat{S}_{(-i) M Q} & =\int_{\Im(\kappa)<-\Im\left(\xi_{N}\right)} d \kappa \frac{1}{2 \sinh \frac{\pi \kappa}{2}} \frac{\sinh \frac{\pi \kappa(2-N)}{4}}{\sinh \frac{\pi \kappa N}{4}} V_{m}^{(2)}(-\kappa) V_{Q}^{(-1)}(\kappa) \\
& +\sum_{\xi= \pm 2 i, 0}(-1)^{\frac{\xi}{2 i}} \tilde{v}_{M}^{(2)}(-\xi) \tilde{V}_{Q}^{(-1)}(\xi) \\
& +i P_{m Q} \\
& =\left(\begin{array}{cc}
z_{i j} & 0 \\
\hat{s}_{(-i) p j} & \hat{S}_{(-i) m q}
\end{array}\right) .
\end{aligned}
$$

\section{The relation between $g h=3$ and $g h=0$}

In the last section we gave the reconstruction formulas for $g h=3$ wedge states. We saw at the beginning that it is not going to be straightforward to write down a corresponding formula

\footnotetext{
${ }^{3}$ In II the states augmented with $z$ were denoted by a prime, $S^{\prime}$. Now we think we can drop the prime without harm.
} 
for $g h=0$ states, the reason being that $g h=0$ Neumann matrices do not (anti)-commute with $G$, so the relevant reconstruction formula cannot involve simply the continuous and discrete eigenvectors of $G$ (no matter whether $\kappa$ is complex, as for all values of $\kappa$ we formally have eigenvectors of $G$ ).

\subsection{The midpoint reflector}

The strategy we are going to follow is thus a bottom up one and it is based on the midpoint 2 -string vertex (reflector). This vertex is the oscillator incarnation of the insertion of $Y$ at the midpoint. Since we have 'two' midpoints (the holomorphic and the anti-holomorphic one), the midpoint insertion will be given by the following reflector(s)

$$
\left\langle V^{(2)}\right|(Y(i), Y(-i))=\left(\left\langle V_{(+i)}^{(2)}\right|,\left\langle V_{(-i)}^{(2)}\right|\right)
$$

where $\left\langle V^{(2)}\right|$ is just the usual 2-string vertex which implements bpz conjugation.

The midpoint reflector is meant to send a $g h=0$ right state (wedge state) to a $g h=3$ left state $(Y(i)$-inserted wedge) as follows

$$
\left(\left\langle V_{(+i)}^{(2)}\left|,\left\langle V_{(-i)}^{(2)}\right|\right) \mid N\right\rangle=\left(\left\langle\hat{N}_{(+i)}\right|,\left\langle\hat{N}_{(-i)}\right|\right)\right.
$$

Each of the two entries is a squeezed state given by

$$
\begin{aligned}
\left\langle V_{( \pm i)}^{(2)}\right| & =\left\langle V^{(2)}\right| Y( \pm i) \\
& ={ }_{12}\left\{\hat{0} \mid \exp \left(-\sum_{n, M} c_{n}^{r} \hat{R}_{( \pm i) n M}^{r s} b_{M}^{s}\right), \quad n \geq 2, M \geq-1, \quad r, s=1,2\right.
\end{aligned}
$$

We concentrate from now on on the $Y(+i)$ insertion, as all the results for the $Y(-i)$ insertion can be obtained by simple complex conjugation. The Neumann coefficients are given by $\left(f_{r}(i)=f_{s}(i)\right)$

$$
\begin{aligned}
\hat{R}_{(i) n M}^{r s} & =\oint_{0} \frac{d z}{2 \pi i} \oint_{0} \frac{d w}{2 \pi i} \frac{1}{z^{m-1}} \frac{1}{w^{N+2}} R_{(i)}^{r s}(z, w) \\
\hat{R}_{(i)}^{r s}(z, w) & =\left\langle Y\left(f_{r, s}(i)\right) f_{r} \circ b(z) f_{s} \circ c(w)\right\rangle-\frac{\delta^{r s}}{z-w} \\
& =\frac{f_{r}^{\prime}(z)^{2}}{f_{s}^{\prime}(w)} \frac{1}{f_{r}(z)-f_{s}(w)}\left(\frac{f_{r}(w)}{f_{s}(z)}\right)^{3}-\frac{\delta^{r s}}{z-w},
\end{aligned}
$$

where the $S L(2, C)$ gluing functions are

$$
f_{r}(z)=(-1)^{r} \frac{1+i z}{1-i z}
$$

The 'principal'/'residual' decomposition is given by

$$
\hat{R}_{(i)}^{r s}(z, w)=\hat{R}^{r s}(z, w)-\frac{4 i}{2}\left((-1)^{r-s} f_{-\frac{4 i}{2}}^{(2)}(z) f_{\frac{4 i}{2}}^{(-1)}(w)+\frac{1}{2} f_{\kappa=0}^{(2)}(z) f_{\kappa=0}^{(-1)}(w)\right),
$$


and the 'principal' part is

$$
\hat{R}^{r s}(z, w)=\left(\frac{2 i}{2} \frac{1+w^{2}}{\left(1+z^{2}\right)^{2}} \frac{f_{r}(z)+f_{s}(w)}{f_{r}(z)-f_{s}(w)}-\frac{\delta^{r s}}{z-w}\right) .
$$

As usual, in addition to the 'principal' part we have 'residual' contributions from $\kappa= \pm \frac{4 i}{2}=$ $\pm 2 i$ and $\kappa=0$. By explicitly computing the Neumann coefficients we get the following matrices

$$
\begin{aligned}
& \hat{R}_{(i)}^{11}=\hat{R}_{(i)}^{22} \equiv \hat{R}_{(i)}=\left(\begin{array}{cc}
0 & 0 \\
r_{n i} & 0
\end{array}\right)=\left(\begin{array}{ll}
0 & 0 \\
r & 0
\end{array}\right) \\
& \hat{R}_{(i)}^{12}=\hat{R}_{(i)}^{21} \equiv \hat{R}_{(i)}^{\prime}=\left(\begin{array}{cc}
0 & 0 \\
(-1)^{n+1} r_{n,-i}^{*} & (-1)^{n} \delta_{n m}
\end{array}\right)=\left(\begin{array}{cc}
0 & 0 \\
-\mathcal{C} r^{*} z & \mathcal{C}
\end{array}\right)
\end{aligned}
$$

The 3-column $r$ is just the Neumann matrix of the $N=2$ wedge (vacuum) with the $Y(+i)$ insertion, this matrix has no bulk but just three columns. Explicitly

$$
\begin{aligned}
r(z, w) & =\sum_{n \geq 2} \sum_{|i| \leq 1} r_{n i} z^{-n+1} w^{-i-2}=\frac{1}{z-w}\left(\frac{w-i}{z-i}\right)^{3}-\frac{1}{z-w} \\
& =t(z, w)-2 i\left(f_{-2 i}^{(2)}(z) f_{2 i}^{(-1)}(w)+\frac{1}{2} f_{\kappa=0}^{(2)}(z) f_{\kappa=0}^{(-1)}(w)\right),
\end{aligned}
$$

Here $t(z, w)$ is the Neumann function of the 'principal' $N=2 g h=3$ wedge.

We now consider the most general $g h=0$ squeezed state

$$
|S\rangle=e^{c_{N}^{\dagger} \mathcal{S}_{N m} b_{m}^{\dagger}}|0\rangle
$$

the matrix $S$ has the structure

$$
\mathcal{S}=\left(\begin{array}{ll}
0 & s \\
0 & S
\end{array}\right)
$$

Now we reflect it by adapting the general method of [53]

$$
\left\langle\hat{S}_{(i)}\right| \equiv\left\langle V_{(i)}^{(2)}|| S\right\rangle=\operatorname{det}\left(1-\mathcal{S} \hat{R}_{(i)}\right)\langle\hat{0}| \exp \left(-c_{n}\left[\hat{R}_{(i)}+\hat{R}_{(i)}^{\prime} \frac{1}{1-\mathcal{S} \hat{R}_{(i)}} \mathcal{S} R_{(i)}^{\prime}\right]_{n M} b_{M}\right)
$$

Using the block decomposition, we have

$$
\frac{1}{1-\mathcal{S} \hat{R}_{(i)}}=\left(\begin{array}{c|c}
\frac{1}{1-s r} & 0 \\
-S r \frac{1}{1-s r} & 1
\end{array}\right)
$$

and

$$
\operatorname{det}\left(1-\mathcal{S} \hat{R}_{(i)}\right)=\operatorname{det}_{3 \times 3}(1-s r)
$$


In total, after some trivial algebra, we get

$$
\begin{gathered}
\hat{R}_{(i)}+\hat{R}_{(i)}^{\prime} \frac{1}{1-\mathcal{S} \hat{R}_{(i)}} \mathcal{S} \hat{R}_{(i)}^{\prime}=\left(\begin{array}{cc}
0 & 0 \\
r-\hat{S}_{(i)} r^{*} z & \hat{S}_{(i)}
\end{array}\right) \\
\hat{S}_{(i)}=\mathcal{C}\left(S+\left(S r-r^{*} z\right) \frac{1}{1-s r} s\right) \mathcal{C}
\end{gathered}
$$

A clarification is in order. We are comparing the Neumann matrix of a ket state, $S$, with the one of a $b r a, \hat{S}_{(i)}$. As we are going to check these relations explicitly for wedge states, this does not cause any problem, because at $g h=0$ we have $\mathcal{C} S \mathcal{C}=S$. However, in view of other possible applications, we should write the above $g h=0 / g h=3$ relation in an unambiguous way, even for $(g h=0)$ states that are not twist invariant. In doing this we have to declare if the squeezed state matrices are the one relative to the bra or the ket representation. Since surface states are usually defined as a bra, we reserve the notation $S$ for the Neumann matrices of bra-states. So, with these conventions, the most general ket-squeezed state is given by

$$
|S\rangle=e^{c_{N}^{\dagger}[\mathcal{C S C}]_{N m} b_{m}^{\dagger}}|0\rangle,
$$

With this convention, using $\mathcal{C} r \mathcal{C}=r^{*}$, the equation (4.16) becomes

$$
\hat{S}_{(i)}=\left(S+\left(S r^{*}-r z\right) \frac{1}{1-s r^{*}} s\right) .
$$

All the matrices entering this equation are the ones which define bra states, both at $g h=0$ and at $g h=3$.

The $Y(-i)$-inserted $g h=3$ Neumann matrix (which is obtained from the reflector $\left\langle V_{(-i)}^{(2)}\right|$ ) will be thus

$$
\hat{S}_{(-i)}=\left(S+\left(S r-r^{*} z\right) \frac{1}{1-s r} s\right) .
$$

\subsection{Midpoint Identities}

The first thing to notice is that when we reflect a $g h=0$ state, the resulting $g h=3$ state will have the leftmost 3 -column that is determined by the $g h=3$ bulk $\hat{S}_{(i)}$, that is all the $g h=3$ squeezed states that we get by reflecting $g h=0$ squeezed states will have a defining matrix of the form

$$
\left(\begin{array}{cc}
0 & 0 \\
\hat{s}_{(i)} & \hat{S}_{(i)}
\end{array}\right),
$$

where the 3-column is given by

$$
\hat{s}_{(i)}=r-\hat{S}_{(i)} r^{*} z
$$


Following the terminology of [50] (where a similar relation for the $g h=1 / g h=2$ doublet was discussed, see also [51, 52]) we call this property midpoint identity.

Since we expect to get the $Y$-inserted $g h=3$ wedges by reflecting the $g h=0$ ones, the first consistency check is to see if the $g h=3$ Neumann functions obey the midpoint identity.

The proof of $g h=3$ midpoint identities is a perfect playground to see the power of reconstruction formulas at work.

It is indeed sufficient to use the previously stated reconstructions, plus the orthogonality relation of the $(-1,2)$ basis

$$
\sum_{n \geq 2} V_{n}^{(-1)}(x) V_{n}^{(2)}(y)=2 \sinh \frac{\pi x}{2} \delta(x-y) .
$$

This orthogonality condition is valid for general complex $(x, y)$, but when $x$ coincides with a pole in the measure $\frac{1}{2 \sinh \frac{\pi x}{2}}$, great care must be exercised.

\subsubsection{Midpoint identities for 'principal' states}

We will first prove a related midpoint identity which links the 3-column and the bulk of the principal $g h=3$ wedges. This will involve the use of the discrete and continuous spectrum, as reviewed above from II. The complex midpoint identity we want to prove will be easily obtained by just parallel-shifting the path of the $\kappa$ integration at $\Im(\kappa)>2$. The 'principal' midpoint identity is

$$
(\hat{S} t z)_{p j}=(t-\hat{s})_{p j} \quad\left(z_{i j}=\delta_{i+j}\right), \quad-1 \leq i, j \leq 1
$$

The short-short matrix $\hat{S}$ is the bulk of the 'principal' $g h=3$ wedge

$$
\hat{S}_{p q}=\int_{-\infty}^{\infty} d \kappa \frac{1}{2 \sinh \frac{\pi \kappa}{2}} \frac{\sinh \frac{\pi \kappa(2-N)}{4}}{\sinh \frac{\pi \kappa N}{4}} V_{p}^{(2)}(-\kappa) V_{q}^{(-1)}(\kappa)
$$

The 3 -column $\hat{s}_{p j}$ is the zero mode part of the 'principal' $g h=3$ wedge (whose reconstruction is given by both the continuous and the discrete spectrum)

$$
\begin{aligned}
\hat{s}_{p j} & =\int_{-\infty}^{\infty} d \kappa \frac{1}{2 \sinh \frac{\pi \kappa}{2}} \frac{\sinh \frac{\pi \kappa(2-N)}{4}}{\sinh \frac{\pi \kappa N}{4}} V_{p}^{(2)}(-\kappa) V_{j}^{(-1)}(\kappa) \\
& +\sum_{\xi= \pm 2 i, 0}(-1)^{\frac{\xi}{2 i}} \tilde{v}_{p}^{(2)}(-\xi) \tilde{V}_{j}^{(-1)}(\xi)
\end{aligned}
$$

The 3 -column $t_{p j}$ is the Neumann matrix of the $N=2$ 'principal' $g h=3$ wedge. In turn the same quantity arises in the 'lame' completeness relation of the continuous and discrete spectrum in the zero mode sector. In particular

$$
t_{n j}=-\int_{-\infty}^{\infty} d \kappa \frac{1}{2 \sinh \frac{\pi \kappa}{2}} V_{n}^{(2)}(\kappa) V_{-j}^{(-1)}(\kappa)=\sum_{\xi= \pm 2 i, 0} \tilde{v}_{n}^{(2)}(\xi) \tilde{V}_{-j}^{(-1)}(\xi) .
$$


We remark that in II this quantity was denoted in a different way,

$$
t_{n j}=-b_{-j n}
$$

A consequence of the above relations is the fact that the zero modes of the $h=-1$ basis are expressible as a linear combination of the non-zero modes

$$
V_{i}^{(-1)}(\kappa)=-V_{n}^{(-1)}(\kappa) t_{n,-i}, \quad \kappa \in R .
$$

Using the above equations it is then immediate to prove (4.21) in the form

$$
\hat{s}_{n i}=(t-\hat{S} t z)_{n i}
$$

\subsubsection{Midpoint identities for $Y(i)$-inserted wedges}

Given the previous result for 'principal' $g h=3$ wedges, we can now easily prove the midpoint identity for the $Y(i)$ insertion

$$
\hat{s}_{(i)}=r-\hat{S}_{(i)} r^{*} z .
$$

To this end it is important to notice that the 3-column $r_{n i}^{*}$ has the same integral representation as $t_{n i}$ but with a shifted path in the complex $\kappa$ plane with $\Im(\kappa)>2^{4}$

$$
r_{n i}^{*}=-\int_{\Im(\kappa)>2} d \kappa \frac{1}{2 \sinh \frac{\pi \kappa}{2}} V_{n}^{(2)}(\kappa) V_{-i}^{(-1)}(\kappa)
$$

This implies that we have

$$
V_{i}^{(-1)}(\kappa)=-V_{n}^{(-1)}(\kappa) r_{n,-i}^{*}, \quad 2<\Im(\kappa)<4
$$

We can now easily compute $\hat{S}_{i} r^{*} z$ by using (4.20) and we get

$$
\begin{aligned}
{\left[\hat{S}_{(i)} r^{*} z\right]_{n j} } & =\int_{\Im(\kappa)>\frac{4}{N}} d \kappa \frac{\mathfrak{t}_{N}(\kappa)}{2 \sinh \frac{\pi \kappa}{2}} V_{n}^{(2)}(-\kappa) V_{m}^{(-1)}(\kappa) \\
& \times\left(-\int_{\Im\left(\kappa^{\prime}\right)>2} d \kappa^{\prime} \frac{1}{2 \sinh \frac{\pi \kappa^{\prime}}{2}} V_{m}^{(2)}\left(\kappa^{\prime}\right) V_{j}^{(-1)}\left(\kappa^{\prime}\right)\right) \\
& =-\int_{\Im\left(\kappa^{\prime}\right)>2} d \kappa^{\prime} \frac{\mathfrak{t}_{N}\left(\kappa^{\prime}\right)}{2 \sinh \frac{\pi \kappa^{\prime}}{2}} V_{m}^{(2)}\left(-\kappa^{\prime}\right) V_{j}^{(-1)}\left(\kappa^{\prime}\right)
\end{aligned}
$$

It is important to notice that, to get the last line of the above equation, we have disregarded the secondary poles of

$$
\mathfrak{t}_{N}(\kappa)=\frac{\sinh \frac{\pi \kappa(2-N)}{4}}{\sinh \frac{\pi \kappa N}{4}},
$$

at $\kappa=n \xi_{N}$ for $n>1$. Starting from the wedge $N=5$ these poles would begin to give contribution when the path is shifted from $\kappa>\xi_{N}$ to $\kappa>2 i$. The reason why they must be neglected is explained in appendix $\mathrm{B}$, where the relevant part of this computation is

\footnotetext{
${ }^{4}$ The same is true for $r_{n i}$ where the path is shifted at $\Im(\kappa)<-2$.
} 
performed in a regularized way on the original $z$-plane.

In order to use the result we got for the 'principal' part ('principal' states) we deform the path back to the real line and we pick up the residues (from just the principal poles) along the way

$$
\int_{\Im\left(\kappa^{\prime}\right)>2}=\int_{R}-\oint_{2 i}-\oint_{\frac{4 i}{N}}-\frac{1}{2} \oint_{0}
$$

which gives (using the midpoint identity for the 'principal' states)

$$
\begin{aligned}
{\left[\hat{S}_{(i)} r^{*} z\right]_{n j}=} & t_{n j}-\hat{s}_{n j}-2 i V_{m}^{(2)}(-2 i) V_{j}^{(-1)}(2 i)+\xi_{N} V_{m}^{(2)}\left(-\xi_{N}\right) V_{j}^{(-1)}\left(\xi_{N}\right) \\
& +\left(\frac{\xi_{N}}{2}-i\right) V_{m}^{(2)}(0) V_{j}^{(-1)}(0) .
\end{aligned}
$$

Remembering now

$$
\begin{aligned}
\hat{s}_{(i) n j} & =\hat{s}_{n j}-\xi_{N}\left(V_{n}^{(2)}\left(-\xi_{N}\right) V_{j}^{(-1)}\left(\xi_{N}\right)+\frac{1}{2} V_{n}^{(2)}(0) V_{j}^{(-1)}(0)\right) \\
r_{n j} & =\left[\left.\hat{s}_{(i)}\right|_{N=2}\right]_{n j}=t_{n j}-2 i\left(V_{n}^{(2)}(-2 i) V_{j}^{(-1)}(2 i)+\frac{1}{2} V_{n}^{(2)}(0) V_{j}^{(-1)}(0)\right),
\end{aligned}
$$

we get finally

$$
\left[\hat{S}_{(i)} r^{*} z\right]_{n j}=\left[r-\hat{s}_{i}\right]_{n j}
$$

Notice that the universal contribution at $\kappa=0$,

$$
-i P_{m j}=-i V_{m}^{(2)}(0) V_{j}^{(-1)}(0)
$$

which comes from the wedge eigenvalue at $\kappa=0$, is there precisely to implement the midpoint identity.

\subsubsection{Midpoint identities for the vertex}

For the 3 -strings vertex defined in paper II $(Y(+i)$-insertion $)$, the midpoint identities easily generalizes to

$$
\hat{v}_{(i)}^{a b}=\delta^{a b} r-\hat{V}_{(i)}^{a b} r^{*} z
$$

To see this we have to compute, using the same strategy as before,

$$
\left[\hat{V}_{(i)}^{a b} r^{*} z\right]_{n i}=-\int_{\Im(\kappa)>2} d \kappa \frac{v^{a b}(\kappa)}{2 \sinh \frac{\pi \kappa}{2}} V_{n}^{(2)}(-\kappa) V_{i}^{(-1)}(\kappa) .
$$

It is important to notice that (see paper II)

$$
v^{a b}(\kappa= \pm 2 i)=\delta^{a b}, \quad v^{a b}(\kappa=0)=\frac{2}{3}-\delta^{a b}
$$


Taking back the path to the real axis

$$
\int_{\Im(\kappa)>2}=\int_{R}-\oint_{2 i}-\oint_{\frac{4 i}{3}}-\frac{1}{2} \oint_{0},
$$

and using

$$
2 \pi i \operatorname{Res}_{\kappa=\frac{4 i}{3}} \frac{v^{a b}(\kappa)}{2 \sinh \frac{\pi \kappa}{2}}=\frac{4 i}{3} \alpha^{a-b}, \quad \alpha=e^{\frac{2 \pi i}{3}}
$$

we get

$$
\begin{aligned}
{\left[\hat{V}_{(i)}^{a b} r^{*} z\right]_{n j}=} & \delta^{a b} t_{n j}-\hat{v}_{n j}^{a b}-2 i \delta^{a b} V_{n}^{(2)}(-2 i) V_{j}^{(-1)}(2 i)+\xi_{3} \alpha^{a-b} V_{n}^{(2)}\left(-\xi_{3}\right) V_{j}^{(-1)}\left(\xi_{3}\right) \\
& +\left(\frac{\xi_{3}}{2}-i \delta^{a b}\right) V_{n}^{(2)}(0) V_{j}^{(-1)}(0) \\
= & {\left[\delta^{a b} r-\hat{v}_{(i)}^{a b}\right]_{n j} }
\end{aligned}
$$

We can repeat the same procedure for the $Y(-i)$-insertion and prove that

$$
\hat{v}_{(-i)}^{a b}=\delta^{a b} r^{*}-\hat{V}_{(-i)}^{a b} r z
$$

These identities will be useful in order to simplify the midpoint product of two $g h=0$ states, as we will see later.

\section{$4.3 g h=0$ from $g h=3$ : zero modes coefficients}

The operator $Y(z)$ has a non trivial kernel so it would seem that, by reflecting a state, some $g h=0$ information will get necessarily lost. This would be the case indeed if we used only one insertion (say $Y(+i)$ ). In order to appreciate this let us concentrate for a moment on the subalgebra of surface states. Let $|f\rangle$ be the surface state associated (modulo $S L(2, C)$ ) to a function $f$, holomorphic on the unit semidisk of the $U H P$. Let then $\left|\hat{f}_{(i)}\right\rangle=Y(i)|f\rangle$ be the $g h=3$ 'dual' of $|f\rangle$. Observing that

$$
b_{-1} b_{0} b_{1} Y(i)|0\rangle=|0\rangle
$$

it follows that

$$
f^{-1} \circ b_{-1} f^{-1} \circ b_{0} f^{-1} \circ b_{1}\left|\hat{f}_{(i)}\right\rangle=|f\rangle .
$$

Now, for $i=-1,0,1$, we have

$$
f^{-1} \circ b_{i}|f\rangle=\left(b_{i}-F_{i m} b_{m}^{\dagger}\right)|f\rangle=0,
$$

where $F_{i m}$ are the zero modes of the Neumann matrix of $|f\rangle$. So we get

$$
\prod_{i=-1,0,1}\left(b_{i}-F_{i m} b_{m}^{\dagger}\right)\left|\hat{f}_{(i)}\right\rangle=|f\rangle
$$

Calling $\hat{F}$ and $F$ the Neumann matrices of $\left|\hat{f}_{(i)}\right\rangle$ and $|f\rangle$ respectively we then get the relation

$$
F_{n m}=\hat{F}_{n m}+\hat{F}_{n,-j} F_{j m}, \quad n, m \geq 2, \quad j=-1,0,1
$$


which is the same relation we get using the midpoint reflector, see below. Notice that, without knowing in advance the zero mode components $F_{i n}$, we cannot go back at $g h=0$. This is a consequence of the fact that $Y$ has a non trivial kernel. ${ }^{5}$

That's the basic reason why the insertions we use are actually two (the holomorphic and the anti-holomorphic midpoint). The simultaneous knowledge of these two $g h=3$ states will be enough to reconstruct the corresponding $g h=0$ squeezed state. We would like now to show how the $g h=0$ zero modes are related to the $g h=3$ Neumann matrices. To this end we notice that, by using the midpoint identity (4.19), the $g h=0 / g h=3$ relations for the $Y(+i)$ insertion, (4.16), can be written as ${ }^{6}$

$$
S-\hat{S}_{(i)}=\hat{s}_{(i)} z s,
$$

while doing the same for the $Y(-i)$ insertion we get $^{7}$

$$
S-\hat{S}_{(-i)}=\hat{s}_{(-i)} z s .
$$

Taking the difference of the two we end up with

$$
\hat{S}_{(i)}-\hat{S}_{(-i)}=\left(\hat{s}_{(-i)}-\hat{s}_{(i)}\right) z s .
$$

Our task is to solve this equation for $s$ ( $g h=0$ zero mode contribution). A quick inspection, (4.16), on the involved degrees of freedom shows that this equation is indeed consistent since it is an equality between two (at most) rank 3 matrices. But we can be more explicit. In case of wedge states (where exact reconstruction formulas are available at $g h=3$ ) it is easy to solve this equation for $s$. For using the known reconstruction formulas, we have ( $\kappa=0$ does not affect the bulk)

$$
\begin{aligned}
\left(\hat{S}_{(i)}-\hat{S}_{(-i)}\right)_{n m} & =-\xi_{N}\left(V_{n}^{(2)}\left(-\xi_{N}\right) V_{m}^{(-1)}\left(\xi_{N}\right)+V_{n}^{(2)}\left(\xi_{N}\right) V_{m}^{(-1)}\left(-\xi_{N}\right)\right) \\
\left(\hat{s}_{(-i)}-\hat{s}_{(i)}\right)_{n j} & =\xi_{N}\left(V_{n}^{(2)}\left(-\xi_{N}\right) V_{j}^{(-1)}\left(\xi_{N}\right)+V_{n}^{(2)}(0) V_{j}^{(-1)}(0)+V_{n}^{(2)}\left(\xi_{N}\right) V_{j}^{(-1)}\left(-\xi_{N}\right)\right),
\end{aligned}
$$

where, as usual, $\xi_{N}=\frac{4 i}{N}$. First, we notice that in order to be able to reproduce (4.39) we take

$$
V_{i}^{(-1)}(0) z_{i j} s_{i m}=V_{-i}^{(-1)}(0) s_{i m}=0
$$

\footnotetext{
${ }^{5}$ It is clear that, if we limit ourselves to surface states, this treatment is kind of overshooting since, for such states, starting from the $g h=3$ Neumann function (2.7), one can adapt the methods of [56] to directly derive the surface-state function $f(z)$. Knowing this function, one can then write down the Neumann coefficients at $g h=0$. However our intent here is to establish a general algebraic procedure to go back at $g h=0$, which can possibly be extended to more general squeezed states.

${ }^{6}$ We are now comparing two bra's so we get rid of the twist matrices in (4.16)

${ }^{7}$ If the $g h=0$ state $|S\rangle$ is twist invariant, then the result from the $Y(-i)$ insertion is obtained by simple twist conjugation of the $Y(i)$ insertion. However, in general, the star product of two twist invariant states $\psi$ and $\phi$ is not twist invariant so the two holomorphic/anti-holomorphic insertions are still needed to get back the star-product $\psi * \phi$ from $Y( \pm i)(\psi * \phi)$.
} 
that is

$$
s_{-1, n}=-s_{1, n}
$$

In this way we recover the fact that $g h=0$ wedge states are annihilated by $b_{1}+b_{-1}$. Now it is easy to see that the only form $s_{i n}$ can take, in order to satisfy (4.39), is

$$
s_{i n}=v_{i}^{(2)}\left(-\xi_{N}\right) V_{m}^{(-1)}\left(\xi_{N}\right)+v_{i}^{(2)}\left(\xi_{N}\right) V_{m}^{(-1)}\left(-\xi_{N}\right),
$$

where $v_{i}^{(2)}\left( \pm \xi_{N}\right)$ are 3-dimensional vectors to be determined by inserting this expression in (4.39). This gives the following orthogonality constraints (which also yield, consistently with our previous assumption, $\left.\left(b_{1}+b_{-1}\right)|N\rangle=0\right)$

$$
\begin{aligned}
V_{-i}^{(-1)}\left( \pm \xi_{N}\right) v_{i}^{(2)}\left(\mp \xi_{N}\right) & =-1 \\
V_{-i}^{(-1)}\left( \pm \xi_{N}\right) v_{i}^{(2)}\left( \pm \xi_{N}\right) & =0 \\
V_{-i}^{(-1)}(0) v_{i}^{(2)}\left( \pm \xi_{N}\right) & =0 .
\end{aligned}
$$

These orthogonality relations completely constrain the $v^{(2)}$ 's to be

$$
v_{j}^{(2)}\left( \pm \xi_{N}\right)=\frac{1}{4}\left(\frac{N^{2}}{4}, \mp \frac{i N}{2},-\frac{N^{2}}{4}\right), \quad j=-1,0,1
$$

One can easily check that, by plugging this in the ansatz (4.42), we exactly reproduce the $s_{i n}$ as defined by the $g h=0$ Neumann function, (2.3). We stress again that this has been possible because of the two complementary insertions of $Y$ in $\pm i$.

\subsubsection{A check: $g h=0$ zero mode coefficients of surface states}

Since the possibility of getting back the $g h=0$ zero modes starting from $g h=3$ data is a key-point of our strategy, we would like to elaborate a little bit on the actual solvability of the equation

$$
\hat{S}_{(i)}-\hat{S}_{(-i)}=\left(\hat{s}_{(-i)}-\hat{s}_{(i)}\right) z s .
$$

By construction this equation can be solved for all $g h=3$ matrices coming from reflecting $g h=0$ ones. This is however an empty statement unless we have a general independent principle to identify a $g h=3$ matrix which is the result of a midpoint reflection. In order to appreciate the problem, let us define

$$
\begin{aligned}
\Gamma_{n m} & \equiv\left[\hat{S}_{(i)}-\hat{S}_{(-i)}\right]_{n m} \\
\gamma_{n j} & \equiv\left[\hat{s}_{(i)}-\hat{s}_{(-i)}\right]_{n j}
\end{aligned}
$$

We are supposed to know these quantities and we have to solve for $s_{i n}$ the equation

$$
\Gamma_{n m}=-\gamma_{n,-j} s_{j m}=-\left(\gamma_{n,-1} s_{1, m}+\gamma_{n, 0} s_{0, m}+\gamma_{n, 1} s_{-1, m}\right)
$$

This is an $\infty \times \infty$ set of equations for the $3 \times \infty$ unknowns $s_{i n}$ so, for a general $\Gamma$, the system is over-constrained and thus without solution. This is obvious: not all $g h=3$ matrices we 
could think of come from $g h=0$ by midpoint reflection. We can try to solve this system by picking three different $n$ 's and calling them $n_{i}$ with $i=-1,0,1$. This choice is not completely free, but it should be done in such a way that

$$
\operatorname{det}_{i j} \gamma_{n_{i}, j} \neq 0
$$

Then, for any $m=2, \ldots, \infty$, we can solve the $3 \times 3$ system

$$
\Gamma_{n_{i}, m}=-\gamma_{n_{i}, j} s_{-j m}
$$

for $s_{j m}$ and get a definite answer. But, in general, the result will depend on the particular choice of the three $n_{i}$ 's. Without extra inputs we don't know under which conditions the whole $\infty \times \infty$ system (4.49) will not be overdetermined.

We can check that the above system is not overdetermined if we restrict to the subalgebra of surface states because in this case we have directly and independently the Neumann functions at $g h=3$. Here too, however, there is a subtlety in the case of projectors because the two midpoints collapse to the same point on the boundary thus giving $\hat{S}_{(i)}=\hat{S}_{(-i)}$. That's not a surprise: projectors are kind of singular objects which should correctly be interpreted as a limit of a sequence of well behaved surface states. For wedge states and the sliver this just means that the $N \rightarrow \infty$ limit should be taken at the very end.

Modulo this subtlety, (4.49) can be explicitly solved for any surface state. In this case we have an explicit expression for $\gamma_{n_{i} j}$ and $\Gamma_{n m}$ from the Neumann function which is just the $b c$ propagator on the surface state geometry in the presence of the midpoint $Y$-insertion. We tried with many different surface states given by almost random holomorphic functions on the disk and (excluding the case of projectors like the sliver, the butterflies, etc...) we always found that, when $\operatorname{det}_{i j} \gamma_{n_{i} j} \neq 0$, the solution of (4.51) for $s_{j m}$ is independent of the random choice of the $n_{i}$ 's (and actually coincides with the $g h=0$ Neumann function). Having found the $s_{j m}$ 's one can then plug them into (4.51) when $\operatorname{det}_{i j} \gamma_{n_{i} j}=0$ and verify consistency.

The strongly constrained linear system we have found above is reminiscent of the fact that surface states are known to satisfy the Hirota equations for the KP hierarchy [54, 55] (or the corresponding hierarchy for the ghost sector, [56]), which implies that the whole Neumann matrix is known once a single row/column is. This leaves us with the possibility that, perhaps, it is only for surface states that the whole picture is consistent. We think that this point deserves further investigation, which, however, would go beyond the scope of this paper; so we leave it as an interesting open problem.

\section{$4.4 g h=0$ from $g h=3$ : bulk reconstruction}

Our strategy is to use the midpoint reflector to relate $g h=0$ Neumann matrices to $g h=3$ ones and hence to infer a spectral decomposition of the former by knowing the one of the 
latter. The reflector gives the $g h=3$ Neumann matrices in terms of the $g h=0$ ones, see (4.17)

$$
\hat{S}_{(i)}=S+\left(S r^{*}-r z\right) \frac{1}{1-s r^{*}} s .
$$

Because of the denominator (which arises from a geometric resummation) this relation is very complicated; also, our logic is to derive the $g h=0$ Neumann matrices by knowing the $g h=3$ ones (whose reconstruction is transparent), so we solve it for $S$ ( $g h=0$ bulk) and get

$$
S=\hat{S}_{(i)}-\left(\hat{S}_{(i)} r^{*}-r z\right) s
$$

This equation is now easy to check analytically using the known reconstruction formulas. In particular for wedge states we can use the relations $(z s=\mathcal{C} s)$ and $\left(\mathcal{C} r \mathcal{C}=r^{*}\right)$ and rewrite $(4.53)$ as

$$
S=\hat{S}_{(i)}-\left(\hat{S}_{(i)}-\mathcal{C}\right) r^{*} s
$$

The reconstruction formulae for all the terms on the right hand side are known and are given by

$$
\begin{gathered}
\hat{S}_{(i) n l}=\int_{\kappa>\xi_{N}} d \kappa \frac{\mathfrak{t}_{N}(\kappa)}{2 \sinh \frac{\pi \kappa}{2}} V_{n}^{(2)}(-\kappa) V_{l}^{(-1)}(\kappa)=\left(\int_{R}-\oint_{\xi_{N}}-\frac{1}{2} \oint_{0}\right)(\ldots), \\
\left(\hat{S}_{i}-\mathcal{C}\right)_{n m}=\int_{\kappa>\xi_{N}} d \kappa \frac{\mathfrak{t}_{N}(\kappa)-1}{2 \sinh \frac{\pi \kappa}{2}} V_{n}^{(2)}(-\kappa) V_{m}^{(-1)}(\kappa) \\
\left(r^{*} s\right)_{m l}=-\int_{\kappa^{\prime}>2 i} d \kappa^{\prime} \frac{V_{m}^{(2)}\left(\kappa^{\prime}\right)}{2 \sinh \frac{\pi \kappa^{\prime}}{2}} \frac{N^{2}}{32}\left(\kappa^{\prime}\left(\kappa^{\prime}+\xi_{N}\right) V_{l}^{(-1)}\left(\xi_{N}\right)+\kappa^{\prime}\left(\kappa^{\prime}-\xi_{N}\right) V_{l}^{(-1)}\left(-\xi_{N}\right)\right),
\end{gathered}
$$

where $R$ represents the real axis.

We recall that

$$
\mathfrak{t}_{N}(\kappa)=\frac{\sinh \frac{\pi \kappa(2-N)}{4}}{\sinh \frac{\pi \kappa N}{4}},
$$

and we have also used

$$
\sum_{i=-1}^{1} V_{-i}^{(-1)}(\kappa) v_{i}^{(2)}\left( \pm \xi_{N}\right)=\frac{N^{2}}{32} \kappa\left(\kappa \mp \xi_{N}\right) .
$$

To see the cancelation of some of the terms we would like to write $\hat{S}_{(i) n l}$ more explicitly as

$$
\hat{S}_{(i) n l}=\int_{R} d \kappa \frac{\mathfrak{t}_{N}(\kappa)}{2 \sinh \frac{\pi \kappa}{2}} V_{n}^{(2)}(-\kappa) V_{l}^{(-1)}(\kappa)-\xi_{N} V_{n}^{(2)}\left(-\xi_{N}\right) V_{l}^{(-1)}\left(\xi_{N}\right)-\frac{1}{2} \oint_{0}(\ldots)
$$

Now, to evaluate the product

$$
\left(\hat{S}_{i}-\mathcal{C}\right)_{n m}\left(r^{*} s\right)_{m l}
$$


we deform the $\kappa^{\prime}>2 i$ contour to $\kappa^{\prime}>\xi_{N}$ and we pick the residue at $\kappa^{\prime}=2 i$ on the way. Since $\mathfrak{t}_{N}(2 i)=1$, this residue is actually vanishing.

Then we can write

$$
\begin{aligned}
\left(\left(\hat{S}_{i}-\mathcal{C}\right) r^{*} s\right)_{n l} & =-\int_{\kappa>\xi_{N}} d \kappa \frac{\left(\mathfrak{t}_{N}(\kappa)-1\right) V_{n}^{(2)}\left(\kappa^{\prime}\right)}{2 \sinh \frac{\pi \kappa^{\prime}}{2}} \\
& \times \frac{N^{2}}{32}\left(\kappa\left(\kappa+\xi_{N}\right) V_{l}^{(-1)}\left(\xi_{N}\right)+\kappa\left(\kappa-\xi_{N}\right) V_{l}^{(-1)}\left(-\xi_{N}\right)\right) .
\end{aligned}
$$

Now we close the contour at infinity, above the line $\kappa=\xi_{N}$, so that the value of this integral will be given by the sum of all the residues at $\kappa=2 i m, \quad m=1,2 \ldots$ and at $\kappa=m \xi_{N}, \quad m=2,3, \ldots$, where we assumed $N>2$. For a reason which will become clear soon we start the summation over the residues at $\kappa=m \xi_{N}$ from $m=1$ and then subtract the $m=1$ contribution. Then we obtain

$$
\begin{aligned}
\left.\left(\hat{S}_{(i)}-\mathcal{C}\right) r^{*} s\right)_{n l}= & \oint \frac{d z}{2 \pi i} \frac{1}{z^{n-1} 2 \xi_{N}^{2}}\left\{\left(-\frac{2}{z^{3}}+\frac{2}{z}-\frac{\xi_{N}}{z^{2}}\right) V_{l}^{(-1)}\left(\xi_{N}\right)\right. \\
& \left.+\left(-\frac{2}{z^{3}}+\frac{2}{z}+\frac{\xi_{N}}{z^{2}}\right) V_{l}^{(-1)}\left(-\xi_{N}\right)\right\} \\
+ & \oint \frac{d z}{2 \pi i} \frac{\xi_{N}\left(1+z^{2}\right)^{-2}}{z^{n-1}}\left\{\frac{e^{2 \xi_{N} \tan ^{-1} z}}{\left(-1+e^{\xi_{N} \tan ^{-1} z}\right)^{3}} V_{l}^{(-1)}\left(\xi_{N}\right)\right. \\
& +\frac{e^{\xi_{N} \tan ^{-1} z}}{\left(-1+e^{\left.\xi_{N} \tan ^{-1} z\right)^{3}} V_{l}^{(-1)}\left(-\xi_{N}\right)\right\}} \\
& -\xi_{N} V_{n}^{(2)}\left(-\xi_{N}\right) V_{l}^{(-1)}\left(\xi_{N}\right),
\end{aligned}
$$

where the last term is the contribution from $m=1$. We also recall that the $\frac{1}{2} \oint_{0}(\ldots)$ of (4.55) does not affect the bulk. Therefore, the right hand side of (4.53) is given by

$$
\begin{aligned}
\left(\hat{S}_{i}-\left(\hat{S}_{i}-\mathcal{C}\right) r^{*} s\right)_{n l}= & -\oint \frac{d z}{2 \pi i} \frac{1}{z^{n-1} 2 \xi_{N}^{2}}\left\{\left(-\frac{2}{z^{3}}+\frac{2}{z}-\frac{\xi_{N}}{z^{2}}\right) V_{l}^{(-1)}\left(\xi_{N}\right)\right. \\
+ & \left.\left(-\frac{2}{z^{3}}+\frac{2}{z}+\frac{\xi_{N}}{z^{2}}\right) V_{l}^{(-1)}\left(-\xi_{N}\right)\right\} \\
& -\oint \frac{d z}{2 \pi i} \frac{\xi_{N}\left(1+z^{2}\right)^{-2}}{z^{n-1}}\left\{\frac{e^{2 \xi_{N} \tan ^{-1} z}}{\left(-1+e^{\xi_{N} \tan ^{-1} z}\right)^{3}} V_{l}^{(-1)}\left(\xi_{N}\right)\right. \\
& +\frac{e^{\xi_{N} \tan ^{-1} z}}{\left(-1+e^{\left.\xi_{N} \tan ^{-1} z\right)^{3}} V_{l}^{(-1)}\left(-\xi_{N}\right)\right\}} \\
& +\int_{R} d \kappa \frac{\mathfrak{t}_{N}(\kappa)}{2 \sinh \frac{\pi \kappa}{2}} V_{n}^{(2)}(-\kappa) V_{l}^{(-1)}(\kappa) .
\end{aligned}
$$

The first term vanishes except for the first three rows. In this case the contribution from this term is exactly canceled by the contribution from the second term. Therefore, in the 
bulk calculation we can drop the first term and we obtain

$$
\begin{aligned}
\left(\hat{S}_{i}-\left(\hat{S}_{i}-\mathcal{C}\right) r^{*} s\right)_{n l}= & -\oint \frac{d z}{2 \pi i} \frac{\xi_{N}\left(1+z^{2}\right)^{-2}}{z^{n-1}}\left\{\frac{e^{2 \xi_{N} \tan ^{-1} z}}{\left(-1+e^{\xi_{N} \tan ^{-1} z}\right)^{3}} V_{l}^{(-1)}\left(\xi_{N}\right)\right. \\
& \left.+\frac{e^{\xi_{N} \tan ^{-1} z}}{\left(-1+e^{\left.\xi_{N} \tan ^{-1} z\right)^{3}}\right.} V_{l}^{(-1)}\left(-\xi_{N}\right)\right\} \\
& +\int_{R} d \kappa \frac{\mathfrak{t}_{N}(\kappa)}{2 \sinh \frac{\pi \kappa}{2}} V_{n}^{(2)}(-\kappa) V_{l}^{(-1)}(\kappa) .
\end{aligned}
$$

This formula is meant to calculate only the bulk of the $g h=0$ wedge state. However, it also gives the correct value of the first three rows, in which case the second term is not contributing and the first term is exactly the $s_{i n}$ we have already obtained.

In total, the reconstruction of $g h=0$ wedge states is given by

$$
S_{M n}=\int_{R} d \kappa \frac{\mathfrak{t}_{N}(\kappa)}{2 \sinh \frac{\pi \kappa}{2}} V_{m}^{(2)}(-\kappa) V_{n}^{(-1)}(\kappa)-\sum_{\xi_{N}= \pm \frac{4 i}{N}} \xi_{N} \mathcal{V}_{M}^{(2)}\left(-\xi_{N}\right) V_{n}^{(-1)}\left(\xi_{N}\right)
$$

The 'exotic' $\mathrm{h}=2$ vector $\mathcal{V}_{M}^{(2)}$ is explicitly given by (its explicit form can be read from (4.59))

$$
\begin{aligned}
\mathcal{V}_{M}^{(2)}\left(\xi_{N}\right) & =\oint_{0} \frac{d z}{2 \pi i} \frac{1}{z^{M-1}} F_{\xi_{N}}^{(2)}(z) \\
F_{\xi_{N}}^{(2)}(z) & =\frac{1}{\left(1+z^{2}\right)^{2}}\left(\frac{1}{1-f_{N}(z)}\right)^{3} f_{N}(z)
\end{aligned}
$$

where $f_{N}(z)$ is the wedge mapping function

$$
f_{N}(z)=\exp \left(\xi_{N} \tan ^{-1} z\right)=\left(\frac{1+i z}{1-i z}\right)^{\frac{2}{N}}
$$

It should be noted that $\mathcal{V}_{M}^{(2)}$, when evaluated at $\xi_{N}= \pm 2 i$, coincides, up to a normalization, with the discrete $v^{(2)}( \pm 2 i)$ eigenvectors of $G$, (3.3).

One can explicitly check that the above reconstruction formula exactly reproduces the Neumann coefficients of $g h=0$ wedges, (2.3).

As an example we show that a sample of the entries for $N=5$ coincide with the residues 
of $(2.3)$

$$
\begin{aligned}
S_{-1,3}^{(N=5)} & =-\sum_{\xi_{5}= \pm \frac{4 i}{5}} \frac{4 i}{5} \mathcal{V}_{-1}^{(2)}\left(-\xi_{5}\right) V_{3}^{(-1)}\left(\xi_{5}\right)=-\frac{7}{25} \\
S_{0,2}^{(N=5)} & =-\sum_{\xi_{5}= \pm \frac{4 i}{5}} \frac{4 i}{5} V_{0}^{(2)}\left(-\xi_{5}\right) V_{2}^{(-1)}\left(\xi_{5}\right)=-\frac{14}{25} \\
S_{1,3}^{(N=5)} & =-\sum_{\xi_{5}= \pm \frac{4 i}{5}} \frac{4 i}{5} V_{1}^{(2)}\left(-\xi_{5}\right) V_{3}^{(-1)}\left(\xi_{5}\right)=+\frac{7}{25} \\
S_{2,2}^{(N=5)} & =\int_{-\infty}^{\infty} d \kappa \frac{\hat{\mathfrak{t}}^{(5)}(\kappa)}{2 \sinh \left(\frac{\pi \kappa}{2}\right)} V_{2}^{(2)}(-\kappa) V_{2}^{(-1)}(\kappa) \\
& -\sum_{\xi_{5}= \pm \frac{4 i}{5}} \xi_{5} V_{2}^{(2)}\left(-\xi_{5}\right) V_{2}^{(-1)}\left(\xi_{5}\right)=-\frac{651}{3125}+\frac{448}{625}=\frac{1589}{3125}
\end{aligned}
$$

Even more amazingly, this reconstruction formula also works for the identity string field $N=1$ (for which we have $\Im\left(\xi_{1}\right)=4>\Im(2 i)$ ), where the same few entries still agrees with (2.3)

$$
\begin{aligned}
S_{-1,3}^{(N=1)} & =-\sum_{\xi_{1}= \pm \frac{4 i}{1}} \frac{4 i}{1} V_{-1}^{(2)}\left(-\xi_{1}\right) V_{3}^{(-1)}\left(\xi_{1}\right)=1 \\
S_{0,2}^{(N=1)} & =-\sum_{\xi_{1}= \pm \frac{4 i}{1}} \frac{4 i}{1} V_{0}^{(2)}\left(-\xi_{1}\right) V_{2}^{(-1)}\left(\xi_{1}\right)=2 \\
S_{1,3}^{(N=1)} & =-\sum_{\xi_{1}= \pm \frac{4 i}{1}} \frac{4 i}{1} V_{1}^{(2)}\left(-\xi_{1}\right) V_{3}^{(-1)}\left(\xi_{1}\right)=-1 \\
S_{2,2}^{(N=1)} & =\int_{-\infty}^{\infty} d \kappa \frac{\hat{\mathfrak{t}}^{(1)}(\kappa)}{2 \sinh \left(\frac{\pi \kappa}{2}\right)} V_{2}^{(2)}(-\kappa) V_{2}^{(-1)}(\kappa) \\
& -\sum_{\xi_{1}= \pm \frac{4 i}{1}} \xi_{1} V_{2}^{(2)}\left(-\xi_{1}\right) V_{2}^{(-1)}\left(\xi_{1}\right)=1+0=1 .
\end{aligned}
$$

This formula (as all the reconstruction formulas in general) is non vanishing also for the ( $i j$ ) block and the ( $n i$ ) 3-column, these parts are however eliminated by the normal ordering on $|0\rangle$.

Perhaps with a slight abuse of language we call (4.60) the spectral representation of the $g h=0$ wedges. With its help it is possible to verify directly the nature of the difficulties met in App.C of II when trying to compute their eigenvalues.

\subsection{Normalization}

In the introduction we defined $g h=0$ and $g h=3$ wedges with no normalization in front (because the normalization would be the one point function of $Y$ on the surface-state geometry). However we have seen that (see section (4.1)) when a $g h=0$ squeezed state 
is reflected to a $g h=3$ one, a normalization is also produced. Since, for squeezed states, BRST invariance implies that the overlap (see appendix A)

$$
<g h=3 \mid g h=0>=1
$$

the appearance of this normalization would seem to ruin everything. But looking closer at this normalization

$$
\mathcal{N}=\operatorname{det}(1-\mathcal{S} R)=\operatorname{det}_{3 \times 3}(1-s r)
$$

it is easy to recognize that this expression is just the overlap of the dual $g h=3$ vacuum $\langle 0| Y(i)$ (whose Neumann matrix is bulkless, just the 3-column $r$ ) with the wedge state $|n\rangle$ (the matter part does not contribute in this case, it is purely ghost business). Now, we check in appendix A that, when universal regularization is used, [57, we have

$$
\mathcal{N}=\langle 0|Y(i)| n\rangle=\left\langle\hat{2}_{i}|| n\right\rangle=1
$$

So we see that, in total, the reflector does not produce any normalization when it acts on a BRST $g h=0$ squeezed state.

This will be valid in general (see [58, 59, 36, 57] for previous, related works on this): The total matter+ghost normalization which is produced by the midpoint star product is globally 1 .

In particular we could generalize our construction to $N$-strings midpoint-vertices, 66], with the expectation that

$$
\left\langle\hat{V}_{(i) N}|| I\right\rangle=\left\langle\hat{V}_{(i) N-1}\right|
$$

will hold with no normalizations in front.

\section{Midpoint multiplication of $g h=0$ squeezed states}

In paper II we saw that if we were allowed to star multiply $g h=3$ matrices (which, upon twisting with the $\mathcal{C}$-matrix are reconstructed by the same upper path as the twisted matrices defined by the vertex), all the matrices in the game would commute and the wedge recursion relations would arise automatically, exactly as for the matter sector. Here we are going to show that, as far as the product matrix is concerned, there is no difference in using $g h=3$ matrices or $g h=0$ ones. This will be a consequence of the midpoint identities and the $g h=0 / g h=3$ relations which we just derived.

In order to avoid heavy computations, we will limit ourselves to ${ }^{8}$

$$
\left\langle\hat{V}_{(i) 3}|| N\right\rangle|0\rangle=\left\langle\widehat{N+1}_{(i)}\right|
$$

Note that we intentionally avoided writing any normalization constant: we are thus assuming that a non-universal matter sector (for example 26 free real bosons) is coupled to

\footnotetext{
${ }^{8}$ The case with two general squeezed states works in exactly the same way.
} 
make $c_{t o t}=0$, ghosts alone cannot be consistent after all. In the absence of normalizations this means

$$
Y(i)(|N\rangle *|0\rangle)=Y(i)|N+1\rangle
$$

The explicit expressions for the midpoint-vertex are (see II)

$$
\begin{aligned}
\left\langle\hat{V}_{(i)}\right| & =\left\langle V_{3}\right| Y(i)=\langle\hat{0}| e^{-c_{n}^{r} \hat{V}_{(i) n M}^{r s} b_{M}^{s}} \\
\hat{V}_{(i)}^{r s}(z, w) & =\left[\frac{\partial f_{3}^{r}(z)^{2}}{\partial f_{3}^{s}(w)} \frac{1}{f_{3}^{r}(z)-f_{3}^{s}(w)}\left(\frac{f_{3}^{s}(w)}{f_{3}^{r}(z)}\right)^{3}-\frac{\delta^{r s}}{z-w}\right] \\
& =\hat{V}^{r s}(z, w)-\frac{4 i}{3}\left(e^{\frac{2 \pi i}{3}(r-s)} f_{-\frac{4 i}{3}}^{(2)}(z) f_{\frac{4 i}{3}}^{(-1)}(w)+\frac{1}{2} f_{\kappa=0}^{(2)}(z) f_{\kappa=0}^{(-1)}(w)\right) \\
& =\int_{\kappa>\xi_{3}} \frac{v_{3}^{r s}(\kappa)}{2 \sinh \frac{\pi \kappa}{2}} f_{-\kappa}^{(2)}(z) f_{\kappa}^{(-1)}(w), \quad \text { only in the bulk. }
\end{aligned}
$$

In passing from the 3 rd to the 4 th row we took the $\kappa$ path above $\Im(\kappa)=\xi_{3}$ and we restricted ourselves just to the bulk, so that we can ignore the discrete spectrum and the $\kappa=0$ contribution.

This, together with the previous properties extracted from the 2-vertex (and from the known properties of the vertex) is all we need. In particular we recall the midpoint identities

$$
\hat{v}_{(i)}^{a b}=\delta^{a b} r-\hat{V}_{(i)}^{a b} r^{*} z
$$

By explicit use of the squeezed states formula, we get (we use a boldface notation to represent (ls) and (sl) matrices and a plain one for their (ss) bulk)

$$
\left\langle\hat{V}_{3(i)}|| N\right\rangle|0\rangle=\mathcal{N} e^{-c_{n}[\mathbf{S} * \mathbf{0}]_{(i)} n M} b_{M},
$$

with

$$
\mathcal{N}=\operatorname{det}\left(1-\hat{\mathbf{V}}_{(i)}^{11} \mathbf{S}\right)
$$

First, since

$$
\hat{\mathbf{V}}_{(i)}^{11}=\left.\hat{\mathbf{S}}_{(i)}\right|_{N=3},
$$

this is just the ghost part of the overlap $\left\langle\hat{3}_{(i)}|| N\right\rangle$ which equals unity, when the matter sector is added and universal regularization is used, see appendix A. As claimed, the vertex does not produce normalizations on BRST invariant states.

Let us now turn to the matrix defining the product, which is given by

$$
[\mathbf{S} \hat{*} \mathbf{0}]_{(i)}=\hat{\mathbf{V}}_{(i)}^{11}+\hat{\mathbf{V}}_{(i)}^{12} \mathbf{S} \frac{1}{1-\hat{\mathbf{V}}_{(i)}^{11} \mathbf{S}} \hat{\mathbf{V}}_{(i)}^{21}
$$


As we did for the reflector, we block decompose the above expression and use the identities (5.5) to get

$$
[\mathbf{S} \hat{*} \mathbf{0}]_{(i)}=\left(\begin{array}{cc}
0 & 0 \\
r-(S \hat{*} 0)_{(i)} r^{*} z & (S \hat{* 0})_{(i)}
\end{array}\right) .
$$

Notice that the vertex automatically implements the midpoint identity for the product state. The product-bulk is given by

$$
(\hat{S * 0})_{(i)}=\hat{V}_{(i)}^{11}+\left(\hat{V}_{(i)}^{12} S+\hat{v}_{(i)}^{12} s\right) \frac{1}{1-\hat{V}_{(i)}^{11} S-\hat{v}_{(i)}^{11} s} \hat{V}_{(i)}^{21} .
$$

Here we see very clearly that, even if $g h=3$ matrices commute among themselves, when also $g h=0$ matrices enter the game, commutativity seems to be lost because, in the product-bulk, we get contributions from the zero modes of the states we multiply. In the absence of a clear relation between $g h=0$ matrices and $g h=3$ ones, we would not be able to proceed further.

But now we can use the results of section 4, in particular the midpoint identities (5.5) and the $g h=3 / g h=0$ relation

$$
S=\hat{S}_{(-i)}-\left(\hat{S}_{(-i)} r-r^{*} z\right) s
$$

which allow us to write

$$
\hat{V}_{(i)}^{12} S+\hat{v}_{(i)}^{12} s=\hat{V}_{(i)}^{12} \hat{S}_{(-i)}(1-r s)
$$

and

$$
\frac{1}{1-\hat{V}_{(i)}^{11} S-\hat{v}_{(i)}^{11} s}=\frac{1}{1-r s} \frac{1}{1-\hat{V}_{(i)}^{11} \hat{S}_{(-i)}} .
$$

Notice that the order of matrices matters in this case as $g h=0$ and $g h=3$ matrices generically do not commute. All in all the product Neumann matrix will just contain $g h=3$ ingredients.

$$
(S \hat{*} 0)_{(i)}=\hat{V}_{(i)}^{11}+\hat{V}_{(i)}^{12} \hat{S}_{(-i)} \frac{1}{1-\hat{V}_{(i)}^{11} \hat{S}_{(-i)}} \hat{V}_{(i)}^{21} .
$$

By twisting the matrices and using $\mathcal{C} S_{(-i)} \mathcal{C}=S_{(i)}$, we finally get

$$
\mathcal{C}(S * 0)_{(i)}=\hat{X}_{(i)}^{11}+\hat{X}_{(i)}^{12} \hat{T}_{(i)} \frac{1}{1-\hat{X}_{(i)}^{11} \hat{T}_{(i)}} \hat{X}_{(i)}^{21} .
$$

This is quite nontrivial: when two gh=0 matrices are midpoint multiplied, they are effectively represented in the star product by $g h=3$ ones.

So, as far as the Neumann matrix is concerned, there is no difference in multiplying $g h=0$ states or 'pure bulk' $g h=0$ states whose Neumann matrix is given by the $g h=3$ one (with 
the opposite $Y$-chirality, since it is a ket).

All we have said till now about the product matrix is formally valid for any squeezed state. But going back to our main interest on wedge states, we see that, if we write the above expression in terms of twisted matrices, only matrices reconstructed by the upper $\kappa^{-}$ path will enter the game, so their product will be regular and hence they will all commute (because they are all reconstructed on the same basis and all the paths are homotopic, in the sense that they can be deformed into one another without crossing singularities). This means that we can substitute matrices with eigenvalues, multiply the eigenvalues and then reconstruct the product matrix with the upper path common to all. Then, from the knowledge of both $(S \hat{*} 0)_{(i)}$ and $(S \hat{*} 0)_{(-i)}$, we can use the results of section 4 to go back to $g h=0$ and thus finally show that

$$
|n\rangle *|m\rangle=|n+m-1\rangle .
$$

We have thus completed our long journey. In particular we have showed that, at the end of the day, everything works as easily as for the zero momentum matter sector where $K_{1}$-invariance directly implies commuting Neumann matrices.

\section{Conclusions and discussion}

Our initial task was to star-multiply two $g h=0$ wedge states in the oscillator formalism. Finally we can claim that we succeeded. To start with we found an explicit regular oscillator definition of the operation

$$
Y( \pm i)(|n\rangle *|m\rangle)=Y( \pm i)|n+m-1\rangle
$$

which in oscillator language (taking the $b p z$ to send ket's into bra's) reads

$$
\left\langle\hat{V}_{( \pm i)}^{(3)}|| n\right\rangle|m\rangle=\left\langle n \widehat{+m-1} 1_{( \pm i)}\right|
$$

That's the only expression we can write in which

- All objects are BRST invariant

- All objects are squeezed states

- The vertex is cyclic in the strings indices

- All objects are annihilated by $K_{1}$

The first property is the most important one as it implies that, if we regulate everything with universal regularization, then the expression (6.2) is true without any normalization in front, so there is no conflict with the CFT method (in which normalizations never enter).

The vertex matrices are not twist invariant but obey a complex twist symmetry whose geometrical meaning is to exchange $Y(i) \leftrightarrow Y(-i)$. We saw that this apparent pathology is 
not a problem at all, since the vertex matrices (in the bulk) are still completely commuting as their reconstruction involves paths in the complex $\kappa$-plane which are all homotopic. Hence dangerous divergences from the poles in the imaginary axis are avoided because they are never crossed (the path is in the " $\kappa$-UHP" for all matrices and it is in the same place for the product). So the complex Neumann coefficients (and hence the lack of twist invariance) are just the result of shifting the path from the real line ('principal' part) to $\Im(\kappa)>\Im\left(\xi_{N}\right)$ ('principal' plus 'residual' part).

While there is no problem of convergence when the paths are homotopic, non-homotopic paths will give rise to divergences, encoded in the explicit appearance of complex delta functions (or, equivalently, in $f_{N}(-i)$ or $f_{N}^{\prime}( \pm i)$ if we use the generating function method on the $z$-plane). The generating function method is actually more trustable because, on the $z$-plane, universal regularization is just our usual branch-point displacement. In all analyzed examples the generating function method shows very clearly that the divergences arise from just the principal poles at $\left(\kappa=-\xi_{N}, 0, \xi_{N}\right)$ and not from secondary poles at $\kappa= \pm n \xi_{N}$. Still it would be desirable to understand this directly on the $\kappa$-plane.

More concretely, we related $g h=3$ and $g h=0$ Neumann matrices by means of the midpoint 2-string vertex which allowed us to uncover many interesting properties.

- All $g h=3$ reflected states obey a midpoint identity which relates the 3 -column of their Neumann matrix to the bulk.

- It is very important to have two complementary reflectors (corresponding to the insertion of $Y( \pm i))$ since this allows us to derive the $g h=0$ zero mode reconstruction by using the known reconstructions at $g h=3$. The elusive zero mode contribution of the $g h=0$ Neumann matrices can be indeed derived by the combined use of the $Y$ insertion at the two midpoints $\zeta= \pm i$. We checked this property for random surface states which are not projectors, finding perfect agreement.

- Once the $g h=0$ zero modes are derived from the $g h=3$ matrices, the bulk part can also be easily reconstructed. This clarifies why we have simple commutation relations at work at $g h=3$, while this is not true at $g h=0$.

- All the potential divergences arising from imaginary poles in the complex $\kappa$ plane are avoided because all the paths in the reconstruction formulas can be deformed into one another without crossing (principal) poles, secondary poles should be ignored and this is independently checked by computing the matrix products on the $z$-plane with universal regularization. Secondary poles are nonetheless needed to go back to $g h=0$ where the 'residual' contributions (which include the complete zero mode sector) are given by summing all of their residues on the imaginary axis.

- The reflector produces a normalization, but this normalization is 1 in universal regularization (since it is just the overlap of the dual vacuum with the wedge state). So, in 
total, the reflector takes a $g h=0$ BRST closed squeezed state with no normalization and gives back a $g h=3$ BRST closed squeezed state with no normalization.

After exploration of the $g h=0 / g h=3$ relation, we finally came to the conclusive point of this paper: in the midpoint product the violation of commutativity at $g h=0$ is elegantly avoided and, in order to do the star product, one can use commuting $g h=3$ matrices instead of $g h=0$ ones. Thus everything works as in the matter sector. After all the initial intuition of I was correct.

\section{Acknowledgments}

C.M. would like to thank Ted Erler for interesting discussions. L.B. would like to thank the GGI Institute in Florence, where part of this research was carried out, for hospitality and financial support. C.M. and D.D.T. would like to thank SISSA for the kind hospitality during part of this research. The work of D.D.T. was supported by the Korean Research Foundation Grant funded by the Korean Government with grant number KRF 2009-0077423. R.J.S.S is supported by CNPq-MCT-Brasil.

\section{Appendix}

\section{A. BRST invariant squeezed states}

It is assumed, [48, 49], that all surface states are BRST invariant when the total central charge vanishes. We will also assume that all surface states with insertions are also BRST invariant, if the insertions are given by BRST invariant primary operators. Example of these operators are $Y(z), c \partial c(z)$ (which are $g h=3 / g h=2$ and purely ghost) or $c(z) V^{(m)}(z)$ (with $V^{(m)}(z)$ a weight 1 primary matter field).

We concentrate from now on on $g h=0$ and $g h=3$ squeezed states, and in particular we zoom on the family of wedge states. This enhances BRST invariance with an extra symmetry, which is $K_{1}=L_{1}+L_{-1}$. Wedge states are indeed the only surface states which are annihilated by $K_{1}$. There can be other $g h=0 / g h=3$ squeezed states which are annihilated by $K_{1}$ but which are not BRST invariant. These states should be considered pathological; examples of these states are $g h=0 / 3$ 'principal' wedges (whose bulk Neumann matrices are just given by the continuous spectrum integrated along the real axis). Such states can be used in intermediate computations because of their very simple structure, but at the end one should add the 'residual' contribution from the $\xi_{N}$ poles in order to restore BRST invariance.

How can we check BRST invariance? The most direct way is to do it by brute force. As a first step one can also check $K_{n}=L_{n}+L_{-n}$ invariance, which is in general anomalous for even $n$ and non-vanishing total central charge. These tests are of course possible, but quite cumbersome. However, while it is difficult to directly check BRST invariance, it is quite easy to disprove it, at least in the $g h=0 / 3$ sector. Indeed the cohomology at $g h=0 / 3$ is one-dimensional, i.e. there is just one state, [61]. The cohomology representative at 
$g h=0$ can be chosen to be the unit operator 1 . The representative at $g h=3$ can be chosen to be $Y(z)$. In terms of states, the $g h=0$ representative is just the $\mathrm{SL}(2, \mathrm{R})$ vacuum

$$
|0\rangle=\mathbf{1}(0)|0\rangle
$$

while at $g h=3$ it is

$$
|\hat{0}\rangle=Y(0)|0\rangle=c_{-1} c_{0} c_{1}|0\rangle .
$$

Since we are focusing on the sector, it is convenient to normalize the space-time volume to $(2 \pi)^{D}$ so that we have

$$
\langle 0|Y(0)| 0\rangle=\left\langle 0\left|c_{-1} c_{0} c_{1}\right| 0\right\rangle=\frac{V}{(2 \pi)^{D}}=1 .
$$

Now consider a pair of $g h=0 / g h=3$ squeezed states in the total matter-ghost CFT

$$
\begin{aligned}
& |f\rangle=\exp \left(\frac{1}{2} a^{\dagger} \cdot F^{(m)} \cdot a^{\dagger}\right) \exp \left(\sum_{N, m} c_{N}^{\dagger} F_{N m} b_{m}^{\dagger}\right)|0\rangle=|0\rangle+(\ldots) \\
& \langle\hat{g}|=\langle\hat{0}| \exp \left(\frac{1}{2} a \cdot G^{(m)} \cdot a\right) \exp \left(-\sum_{n, M} c_{n} G_{n M} b_{M}\right)=\langle\hat{0}|+(\ldots)
\end{aligned}
$$

If these two squeezed states are BRST invariant, it means that the (...) are $Q$-exact (precisely because the two vacua exhaust the $g h=0 / 3$ cohomology). Then we are lead to conclude that $\left(c_{t o t}=0\right.$, otherwise the notion of cohomology is not even defined)

$$
\langle\hat{g}|| f\rangle=\left\langle 0\left|c_{-1} c_{0} c_{1}\right| 0\right\rangle=1
$$

That is: BRST invariance implies unit scalar product for any pair of BRST invariant $g h=0 / 3$ squeezed states.

Reversing this property: If $|f\rangle$ and $\langle\hat{g}|$ are two $g h=0 / 3$ squeezed states and $\langle\hat{g}|| f\rangle \neq 1$, then at least one of the two states is not BRST invariant.

We can thus use this property to show that some of the squeezed states which can be built using the $K_{1}$ basis are not $\mathrm{Q}$-closed. In fact, in the oscillator language the quantity $\langle\hat{g} \| f\rangle$ is given by (bosons at the denominator, fermions at the numerator)

$$
\langle\hat{g} \| f\rangle=\frac{\operatorname{det}\left(1-G_{s l} F_{l s}\right)}{\operatorname{det}\left(1-G^{(m)} \cdot F^{(m)}\right)^{\frac{D}{2}}}=\frac{\operatorname{det}\left(1-F_{l s} G_{s l}\right)}{\operatorname{det}\left(1-G^{(m)} \cdot F^{(m)}\right)^{\frac{D}{2}}}
$$

If this quantity can be computed and turns out not to be 1 , it means that (at least) one of the states is not BRST invariant. In practice this ratio is very delicate, and when it is not 1 it is vanishing or diverging (for Neumann matrices of infinite rank). One thus needs a trustable regularization procedure to control this norm. This very non trivial regularization was put on a firm ground by Fuchs and Kroyter, under the name of universal regularization, [57. The idea is very simple: shrink the string field a little bit with the operator $e^{s L_{0}}$ with $s \rightarrow 1^{-}$in order to detach it from the midpoint while computing the overlaps. We actually 
used this regularization when computing the product of infinite matrices on the $z$-plane in II.

In this particular case this regularization consists in regulating the (matter and ghost) matrices in the following universal (the same for matter and ghosts) way

$$
\begin{aligned}
F_{n m}^{(m)} & \rightarrow s^{n+m} F_{n m}^{(m)} \\
G_{n m}^{(m)} & \rightarrow s^{n+m} G_{n m}^{(m)} \\
F_{N m} & \rightarrow s^{N+m} F_{N m} \\
G_{n M} & \rightarrow s^{n+M} G_{n M},
\end{aligned}
$$

where $s \rightarrow 1^{-}$.

For large $(\mathrm{nm})$ this regularization has the effect of truncating with an exponential cutoff, which is stronger than any other power-law divergence which one can encounter. So, as far as $s<1$, the Neumann matrices will be effectively truncated to a finite level. Let us give the relation between the $s$ regulator and level truncation. Using wedge states we empirically found

$$
1-s \sim \frac{2}{L}
$$

This relation should be understood in the following way:

- Pick an $s<1$ and compute numerically (A.3) by using the regularized matrices. The numerical computation can only be done with finite size matrices, so one has to truncate all the matrices to a level $L$ (the same for matter and ghost). The result will be finite.

- One increases the level by keeping the same $s$, the exp-cutoff given by $s$ will assure convergence to a finite value as $L \rightarrow \infty$. The limiting value will be almost given by a finite level $L \sim \frac{2}{1-s}$, after this level corrections will become very small.

- Pick another $s$ which is closer to 1 than the previous one. Repeat the above procedure.

- The closer is $s$ to 1 , the higher should be the level in order to see a convergence pattern.

It is clear that one would like to be able to compute (A.3) analytically in the parameter $s$ : this is certainly doable by generalizing the techniques of [57 (which deal with bosonic ghosts) to our case. In any case, even within a numerical approach, this procedure gives very unambiguous results which mark a sharp distinction between BRST invariant squeezed states and the others.

\section{A.1 BRST invariant $g h=0 / 3$ wedges}

Wedge states are given by ( $g h=0$, matter plus ghosts)

$$
|n\rangle=e^{\frac{1}{2} a_{p}^{\dagger} \cdot M_{p q}^{(n)} \cdot a_{q}^{\dagger}} e^{c_{N}^{\dagger} S_{N m}^{(n)} b_{m}^{\dagger}}|0\rangle
$$


with the defining matrices given by

$$
\begin{aligned}
& M_{p q}^{(n)}=\frac{1}{\sqrt{p q}} \oint_{0} \frac{d z}{2 \pi i} \oint_{0} \frac{d w}{2 \pi i} \frac{1}{z^{p}} \frac{1}{w^{q}}\left[\frac{f_{n}^{\prime}(z) f_{n}^{\prime}(w)}{f_{n}(z)-f_{n}(w)}-\frac{1}{(z-w)^{2}}\right] \\
& S_{N m}^{(n)}=\oint_{0} \frac{d z}{2 \pi i} \oint_{0} \frac{d w}{2 \pi i} \frac{1}{z^{N-1}} \frac{1}{w^{m+2}}\left[\frac{f_{n}^{\prime}(z)^{2}}{f_{n}^{\prime}(w)} \frac{1}{f_{n}(z)-f_{n}(w)}\left(\frac{f_{n}(w)-f_{n}(0)}{f_{n}(z)-f_{n}(0)}\right)^{3}-\frac{w^{3}}{z^{3}(z-w)}\right]
\end{aligned}
$$

We now want to consider $g h=3$ wedges. There is an infinite family of them, as they differ in the location where one inserts the operator $Y(z)$. Out of the infinite places where one can insert $Y$, it is only the midpoint which is consistent with $K_{1}$ invariance. If we want to have a single squeezed state we have two choices, $z= \pm i$

$$
\begin{aligned}
& \left\langle\hat{n}_{(+i)}\right|=\langle n| Y(+i)=\langle\hat{0}| e^{\frac{1}{2} a_{p} \cdot M_{p q}^{(n)} \cdot a_{q}} e^{-c_{m} \hat{S}_{(i)}^{(n)}{ }_{m N} b_{N}} \\
& \left\langle\hat{n}_{(-i)}\right|=\langle n| Y(-i)=\langle\hat{0}| e^{\frac{1}{2} a_{p} \cdot M_{p q}^{(n)} \cdot a_{q}} e^{-c_{m} \hat{S}_{(-i)}^{(n)} m N} b_{N}
\end{aligned}
$$

The only change wrt $g h=0$ is in the ghost Neumann matrices, 2.11, 2.12).

To give an example, we plot in figure 1 the numerical evaluation of $\left\langle\hat{n}_{(i)}|| n\right\rangle$ for $n=3$ using (A.3).
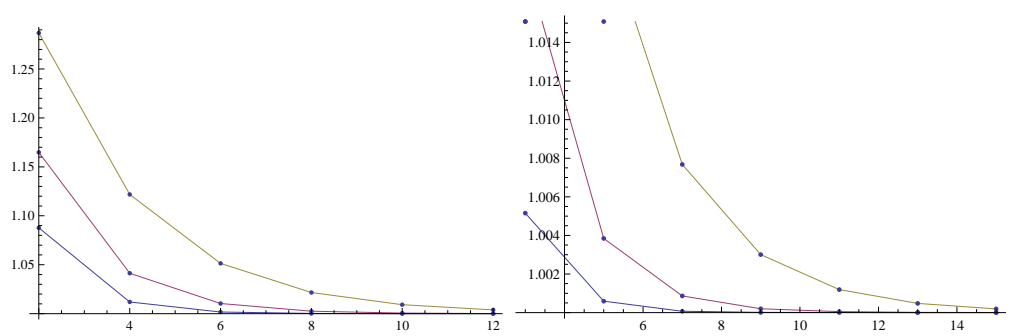

Figure 1: Evaluation of the overlap $\left\langle\hat{3}_{(i)} \| 3\right\rangle$ for even (on the left) and odd (on the right) levels. The horizontal axis is the level (same for the matter and ghosts), while the vertical axis is $\left\langle\hat{3}_{(i)} \| 3\right\rangle$. Three values of the universal regulator $s$ are shown, $s=0.6,0.7,0.8: s=0.6$ corresponds to the set of points which (level by level) are closer to the value $\left\langle\hat{3}_{(i)} \| 3\right\rangle=1$, while $s=0.8$ corresponds to the most distant set. Notice that, as $s$ gets closer to 1 , the level should be increased in order to have a good convergence to the expected unit value and that a reliable level is given by $L \sim 2 /(1-s)$.

It is easy to check that all the other overlaps with different wedges confirm that

$$
\left\langle\hat{n}_{( \pm i)}|| m\right\rangle=1, \quad \forall n, m \geq 1, \quad \forall s<1 .
$$

It is a quite encouraging result, which has to do with the self-consistency of the oscillator formalism with fermionic ghosts. More on this is currently under investigation, [60].

As a counter-example, it is easy to check that, using the 'principal' $g h=3$ wedges, instead of the BRST invariant ones, the norm (for fixed $s<1$ ) will still converge to a finite value (although not the same for even and odd levels), but, as $s$ approaches 1 the limit is more and more distant from unity : this reveals a BRST breakdown. See figure 2 for an example. 


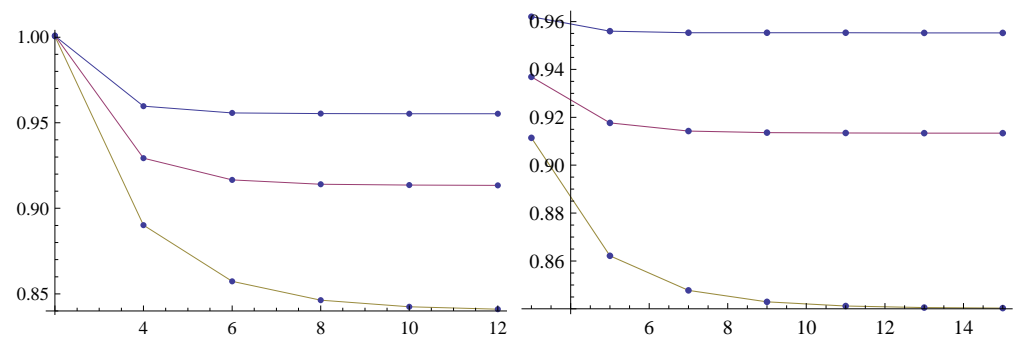

Figure 2: Evaluation of the overlap $\langle\hat{3}|| 3\rangle$ for even (on the left) and odd (on the right) levels, using principal wedges instead of $Y( \pm i)$-inserted one as $g h=3$ duals. The three different sets of points are given by $s=0.6,0.7,0.8: s=0.6$ corresponds to the highest set of points in the plot, while $s=0.8$ corresponds to the lowest set. Notice that there is a convergence pattern for any value of $s<1$, but the limit gets far from unity as $s$ approaches 1 .

\section{B. Irrelevance of secondary poles}

In this appendix we would like to show that secondary poles should not be considered when the product of two $g h=3$ matrices is performed. The way we are going to show this is via an independent computation. We take as an example the proof of the midpoint identity

$$
\hat{S}_{(i)} r^{*} z=r-\hat{s}_{(i)},
$$

similar computations can be performed for all other products of $g h=3$ matrices which are considered in the main text.

First of all, let us trace the point where secondary poles would give a contribution in the derivation of the above equation using the reconstruction formulas. We have

$$
\left[\hat{S}_{(i)} r^{*} z\right]_{n j}=-\int_{\Im\left(\kappa^{\prime}\right)>2} d \kappa^{\prime} \int_{\Im(\kappa)>\xi_{N}} d \kappa \frac{\mathfrak{t}_{N}(\kappa)}{2 \sinh \frac{\pi \kappa^{\prime}}{2}} V_{n}^{(2)}(-\kappa) V_{j}^{(-1)}\left(\kappa^{\prime}\right) \delta\left(\kappa-\kappa^{\prime}\right)
$$

In the main text we just assumed that, given $\xi_{N}<2 i$, we could always take the $\kappa$-path to $\kappa>2 i$, because there is no pole at $\kappa=2 i$. However, in the $\kappa-\mathrm{UHP}$, the wedge eigenvalue $\mathfrak{t}_{N}(\kappa)$ have poles at $\kappa=n \xi_{N}$ for all positive integers $n$ 's. We call the $n=1$ pole a principal pole, while we call the others secondary poles. The apparent problem here is that, starting from $N>4$, some secondary poles will be below $\Im(\kappa)=2$. So they should be picked up when we shift the $\kappa$ path from $\kappa>\xi_{N}$ to $\kappa>2 i$.

Taking into account the contribution from secondary poles we would have ended with

$$
\left[\hat{S}_{(i)} r^{*} z\right]_{n j}=\left[r-\hat{s}_{(i)}\right]_{n j}-4 i \xi_{N} \sum_{k=2}^{n^{*}} \sinh \frac{\pi k \xi_{N}}{2} \delta\left(k \xi_{N}-2 i\right) V_{n}^{(2)}\left(-k \xi_{N}\right) V_{j}^{(-1)}(2 i),
$$

where $n^{*}$ is the maximal integer for which $\frac{4}{N} n^{*}<2$. Notice the naked complex delta contribution which denounces an anomalous divergent part. In the main text we have assumed that one need not consider this contribution. 
We want to show here that what we did is correct and it is what is implied by universal regularization. The way we are going to show it is via an independent computation which, instead of using the power of reconstruction formulas, explicitly performs the matrix product via generating functions defined on the "Fourier transform" of the $\kappa$ plane, the original $z$-plane (that is by the method extensively used in II). In doing this we explicitly consider the 'principal' + 'residual' decomposition

$$
\begin{aligned}
\hat{S}_{(i) n m} & =\oint_{0} \frac{d z}{2 \pi i} \oint_{0} \frac{d w}{2 \pi i} \frac{1}{z^{n-1}} \frac{1}{w^{m+2}}\left(\hat{S}(z, w)-\xi_{N} f_{-\xi_{N}}^{(2)}(z) f_{\xi_{N}}^{(-1)}(w)\right) \\
& =\hat{S}_{n m}-\xi_{N} V_{n}^{(2)}\left(-\xi_{N}\right) V_{m}^{(-1)}\left(\xi_{N}\right),
\end{aligned}
$$

and

$$
\begin{aligned}
r_{m j}^{*} & =\oint_{0} \frac{d z}{2 \pi i} \oint_{0} \frac{d w}{2 \pi i} \frac{1}{z^{m-1}} \frac{1}{w^{j+2}}\left(t(z, w)+2 i f_{2 i}^{(2)}(z) f_{-2 i}^{(-1)}(w)+i f_{0}^{(2)}(z) f_{0}^{(-1)}(w)\right) \\
& =t_{m j}+2 i V_{m}^{(2)}(2 i) V_{j}^{(-1)}(-2 i)+i V_{m}^{(2)}(0) V_{j}^{(-1)}(0) .
\end{aligned}
$$

The terms which are going to contribute to our problem are

$$
\sum_{m \geq 2}\left(-\xi_{N} V_{n}^{(2)}\left(-\xi_{N}\right) V_{m}^{(-1)}\left(\xi_{N}\right)\right)\left(2 i V_{m}^{(2)}(2 i) V_{j}^{(-1)}(-2 i)\right)
$$

and

$$
\sum_{m \geq 2} \hat{S}_{n m}\left(2 i V_{m}^{(2)}(2 i) V_{j}^{(-1)}(-2 i)\right)
$$

Let us begin by computing the inner product

$$
V_{m}^{(-1)}\left(\xi_{N}\right) V_{m}^{(2)}(2 i)
$$

Using the continuous basis we get the formal expression

$$
V_{m}^{(-1)}\left(\xi_{N}\right) V_{m}^{(2)}(2 i)=2 \sinh \frac{\pi \xi_{N}}{2} \delta\left(\xi_{N}-2 i\right)
$$

Since $\xi_{N} \neq 2 i$, one would be tempted to take $\delta\left(\xi_{N}-2 i\right)=0$, this is however not consistent with the explicit result we get by computing the same quantity on the $z$ plane $^{9}$

$$
V_{m}^{(-1)}\left(\xi_{N}\right) V_{m}^{(2)}(2 i)=\oint_{0} \frac{d z}{2 \pi i} \oint_{0} \frac{d w}{2 \pi i} \frac{1}{z^{m+2}} \frac{1}{w^{m-1}} \frac{1+z^{2}}{\left(1+w^{2}\right)^{2}} f_{N}(z)\left(\frac{1+i w}{1-i w}\right)
$$

where

$$
f_{N}(z)=\left(\frac{1+i z}{1-i z}\right)^{\frac{2}{N}} .
$$

As we saw in II, in order to sum up the geometric series, we have to regulate the wedgefunction $\left(\frac{1+i z}{1-i z}\right)^{\frac{2}{N}}$ by pushing the branch points away from $z= \pm i$. As usual we do this by

$$
f_{N}(z) \rightarrow f_{N}^{(K)}(z) \equiv f_{N}(z / K)
$$

\footnotetext{
${ }^{9}$ Also, from general properties of analytic continuation, $\delta(i x)$ is a formal divergent quantity, for $x \in R$.
} 
Then, as argued in II (there are no poles at $z=w$ ), in the integration, we can choose the ordering

$$
\frac{1}{K}<\frac{1}{z}<w<1<\frac{1}{w}<z<K
$$

and, by performing the integral around $w$, we end up with

$$
V_{m}^{(-1)}\left(\xi_{N}\right) V_{m}^{(2)}(2 i)=\oint_{i} \frac{d z}{2 \pi i} \frac{1}{(z-i)^{2}} f_{N}^{(K)}(z)=\left.\frac{d}{d z} f_{N}^{(K)}(z)\right|_{z=i}
$$

which is divergent. So, calling this divergence $\gamma$ we can make the association,

$$
\left.\gamma \equiv \frac{d}{d z} f_{N}^{(K)}(z)\right|_{z=i} \longleftrightarrow 2 \sinh \frac{\pi \xi_{N}}{2} \delta\left(\xi_{N}-2 i\right)
$$

Finally we have

$$
-\xi_{N} V_{n}^{(2)}\left(-\xi_{N}\right) V_{m}^{(-1)}\left(\xi_{N}\right) 2 i V_{m}^{(2)}(2 i) V_{j}^{(-1)}(-2 i)=-2 i \xi_{N} \gamma V_{n}^{(2)}\left(-\xi_{N}\right) V_{j}^{(-1)}(-2 i)
$$

This anomalous contribution needs to be compensated (and will) by the other term we consider,

$$
\sum_{m \geq 2} \hat{S}_{n m}\left(2 i V_{m}^{(2)}(2 i) V_{j}^{(-1)}(-2 i)\right) .
$$

Here we need to compute

$$
\hat{S}_{n m} V_{m}^{(2)}(2 i)
$$

Again, we will first compute this quantity on the un-regularized $\kappa$-plane (that is with the techniques of reconstruction formulas). This gives

$$
\begin{aligned}
\hat{S}_{n m} V_{m}^{(2)}(2 i) & =\mathfrak{t}_{N}(2 i) V_{n}^{(2)}(-2 i)+2 \xi_{N} \sinh \frac{\pi \xi_{N}}{2} V_{n}^{(2)}\left(-\xi_{N}\right) \delta\left(\xi_{N}-2 i\right) \\
& +2 \xi_{N} \sum_{k=2}^{n^{*}} \sinh \frac{\pi k \xi_{N}}{2} V_{n}^{(2)}\left(-k \xi_{N}\right) \delta\left(k \xi_{N}-2 i\right)
\end{aligned}
$$

this expression is what one gets by taking the $\kappa$ path of $S_{n m}$ from the real axis to $\Im(\kappa)=2$, and accordingly picking up the residues along the way. Notice the contribution from the principal pole at $\kappa=\xi_{N}$ (principal divergence), as well as from the secondary ones at $\kappa=k \xi_{N}$ (secondary divergences). The divergence given by the principal pole exactly cancels with the previous term, but secondary poles cannot be canceled against anything else.

We now show that, doing the same computation on the regularized $z$-plane (that is pointsplitting plus contour integral techniques of section 2 of II), there will be no contribution at all from secondary poles. Explicitly (we do not write down the radial ordering term $\frac{1}{z-w}$ in $\hat{S}_{n m}$, because we are just interested in extracting midpoint singularities)

$$
\begin{aligned}
\left.\hat{S}_{n m} V_{m}^{(2)}(2 i)\right|_{\text {sing }}= & \oint_{0} \frac{d z}{2 \pi i} \oint_{0} \frac{d w}{2 \pi i} \oint_{0} \frac{d x}{2 \pi i} \frac{1}{z^{n-1}} \frac{1}{w^{m+2}} \frac{1}{x^{m-1}} \\
& \frac{\xi_{N}}{2} \frac{1+w^{2}}{\left(1+z^{2}\right)^{2}} \frac{f_{N}(z)+f_{N}(w)}{f_{N}(z)-f_{N}(w)} \frac{1}{\left(1+x^{2}\right)^{2}}\left(\frac{1+i x}{1-i x}\right) .
\end{aligned}
$$


Again, in order to perform the sum over $m$, we regulate the wedge function

$$
f_{N}(w) \rightarrow f_{N}^{(K)}(w) \equiv f_{N}(w / K)
$$

and take

$$
\frac{1}{K}<\frac{1}{w}<x<1<\frac{1}{x}<w<K
$$

We can now safely integrate over $x$ and get

$$
\begin{aligned}
& =\frac{\xi_{N}}{2} \oint_{0} \frac{d z}{2 \pi i} \frac{1}{z^{n-1}} \frac{1}{\left(1+z^{2}\right)^{2}} \oint \frac{d w}{2 \pi i} \frac{1}{(w-i)^{2}} \frac{f_{N}(z)+f_{N}^{(K)}(w)}{f_{N}(z)-f_{N}^{(K)}(w)} \\
& =\frac{\xi_{N}}{2} \oint_{0} \frac{d z}{2 \pi i} \frac{1}{z^{n-1}} \frac{1}{\left(1+z^{2}\right)^{2}} \frac{d}{d w}\left(\frac{f_{N}(z)+f_{N}^{(K)}(w)}{f_{N}(z)-f_{N}^{(K)}(w)}\right)_{w=i} \\
& =\left.\frac{\xi_{N}}{2} \oint_{0} \frac{d z}{2 \pi i} \frac{1}{z^{n-1}} \frac{1}{\left(1+z^{2}\right)^{2}} \frac{2}{f_{N}(z)} \frac{d}{d w} f_{N}^{(K)}(w)\right|_{w=i} \\
& =\xi_{N} \gamma V_{n}^{(2)}\left(-\xi_{N}\right)=2 \xi_{N} \sinh \frac{\pi \xi_{N}}{2} V_{n}^{(2)}\left(\xi_{N}\right) \delta\left(\xi_{N}-2 i\right)=\left.\hat{S}_{n m} V_{m}^{(2)}(2 i)\right|_{s i n g} .
\end{aligned}
$$

In integrating over $w$ we just considered the pole at $w=i$, because it is the only one which is within the integration contour.

As claimed, the regularized computation on the $z$-plane (which is of course more trustable than the un-regularized computation on the $\kappa$ plane) clearly shows that secondary poles should not be considered. A clear $\kappa$-plane argument for disregarding them should emerge by the use of universal regularization, [57], (whose basic effect, on the $z-$ plane, is just our branch-points displacement). We leave this quite technical point for future investigations.

Similar checks can be done in all the other computations in the core of the paper: when two $g h=3$ (wedge) matrices are multiplied, only the poles at $\kappa=-\xi_{N}, 0, \xi_{N}$ have to be considered and the homotopy class of the paths in the game will be always understood to be the one implied by these principal poles.

\section{References}

[1] L. Bonora, C. Maccaferri, R. J. Scherer Santos and D. D. Tolla, Ghost story. I. Wedge states in the oscillator formalism, arXiv:0706.1025 [hep-th].

[2] L. Bonora, C. Maccaferri, R. J. Scherer Santos and D. D. Tolla, Ghost story. II. The midpoint ghost vertex, arXiv:0908.0055 [hep-th].

[3] L. Bonora, R. J. Scherer Santos and D. D. Tolla, Spectral properties of ghost Neumann matrices, Phys. Rev. D 77 (2008) 106001 [arXiv:0801.2099 [hep-th]].

[4] E. Witten, "Noncommutative Geometry And String Field Theory," Nucl. Phys. B 268 (1986) 253.

[5] L. Rastelli and B. Zwiebach, "Tachyon potentials, star products and universality," JHEP 0109 (2001) 038 [arXiv:hep-th/0006240]. 
[6] L. Rastelli, A. Sen and B. Zwiebach, "Boundary CFT construction of D-branes in vacuum string field theory," JHEP 0111 (2001) 045 [arXiv:hep-th/0105168].

[7] M. Schnabl, "Wedge states in string field theory," JHEP 0301 (2003) 004 [arXiv:hep-th/0201095].

[8] M. Schnabl, Analytic solution for tachyon condensation in open string field theory, Adv. Theor. Math. Phys. 10 (2006) 433 [arXiv:hep-th/0511286].

[9] Y. Okawa, Comments on Schnabl's analytic solution for tachyon condensation in Witten's open string field theory, JHEP 0604 (2006) 055 [arXiv:hep-th/0603159].

[10] E. Fuchs and M. Kroyter, On the validity of the solution of string field theory, JHEP 0605 (2006) 006 [arXiv:hep-th/0603195].

[11] I. Ellwood and M. Schnabl, Proof of vanishing cohomology at the tachyon vacuum, JHEP 0702 (2007) 096 [arXiv:hep-th/0606142].

[12] L. Rastelli and B. Zwiebach, Solving open string field theory with special projectors, arXiv:hep-th/0606131.

[13] Y. Okawa, L. Rastelli and B. Zwiebach, Analytic solutions for tachyon condensation with general projectors, arXiv:hep-th/0611110.

[14] T. Erler and M. Schnabl, "A Simple Analytic Solution for Tachyon Condensation," arXiv:0906.0979 [hep-th].

[15] T. Erler, "Tachyon Vacuum in Cubic Superstring Field Theory," JHEP 0801 (2008) 013 [arXiv:0707.4591 [hep-th]].

[16] I. Y. Aref'eva, R. V. Gorbachev and P. B. Medvedev, "Tachyon Solution in Cubic Neveu-Schwarz String Field Theory," Theor. Math. Phys. 158 (2009) 320 [arXiv:0804.2017 [hep-th]].

[17] Y. Okawa, Analytic solutions for marginal deformations in open superstring field theory, arXiv:0704.0936 [hep-th].

[18] Y. Okawa, Real analytic solutions for marginal deformations in open superstring field theory, arXiv:0704.3612 [hep-th].

[19] M. Schnabl, Comments on marginal deformations in open string field theory, arXiv:hep-th/0701248.

[20] M. Kiermaier, Y. Okawa, L. Rastelli and B. Zwiebach, Analytic solutions for marginal deformations in open string field theory, arXiv:hep-th/0701249.

[21] E. Fuchs, M. Kroyter and R. Potting, Marginal deformations in string field theory, arXiv:0704.2222 [hep-th].

[22] B. H. Lee, C. Park and D. D. Tolla, "Marginal Deformations as Lower Dimensional D-brane Solutions in Open String Field theory," arXiv:0710.1342 [hep-th].

[23] O. K. Kwon, "Marginally Deformed Rolling Tachyon around the Tachyon Vacuum in Open String Field Theory," Nucl. Phys. B 804 (2008) 1 [arXiv:0801.0573 [hep-th]].

[24] M. Kiermaier and Y. Okawa, "General marginal deformations in open superstring field theory," arXiv:0708.3394 [hep-th]. 
[25] M. Kiermaier and Y. Okawa, "Exact marginality in open string field theory: a general framework," arXiv:0707.4472 [hep-th].

[26] T. Erler, "Marginal Solutions for the Superstring," JHEP 0707 (2007) 050 [arXiv:0704.0930 [hep-th]].

[27] E. Fuchs and M. Kroyter, Schnabl's L(0) operator in the continuous basis, JHEP 0610 (2006) 067 [arXiv:hep-th/0605254].

[28] M. Kroyter, "On string fields and superstring field theories," arXiv:0905.1170 [hep-th].

[29] M. Kroyter, "Comments on superstring field theory and its vacuum solution," arXiv:0905.3501 [hep-th].

[30] I. Ellwood, "Singular gauge transformations in string field theory," arXiv:0903.0390 [hep-th].

[31] M. Kiermaier, Y. Okawa and B. Zwiebach, "The boundary state from open string fields," arXiv:0810.1737 [hep-th].

[32] I. Ellwood, "The closed string tadpole in open string field theory," JHEP 0808 (2008) 063 [arXiv:0804.1131 [hep-th]].

[33] E. Fuchs and M. Kroyter, Analytical Solutions of Open String Field Theory, arXiv:0807.4722 [hep-th].

[34] K. Furuuchi and K. Okuyama, "Comma vertex and string field algebra," JHEP 0109 (2001) 035 [arXiv:hep-th/0107101].

[35] I. Kishimoto, Some properties of string field algebra, JHEP 0112 (2001) 007 [arXiv:hep-th/0110124].

[36] D. M. Belov, Witten's ghost vertex made simple (bc and bosonized ghosts), Phys. Rev. D 69 (2004) 126001 [arXiv:hep-th/0308147].

[37] D.J.Gross and A.Jevicki, Operator Formulation of Interacting String Field Theory, Nucl.Phys. B283 (1987) 1.

[38] D.J.Gross and A.Jevicki, Operator Formulation of Interacting String Field Theory, 2, Nucl.Phys. B287 (1987) 225.

[39] E.Cremmer,A.Schwimmer, C.Thorn, "The vertex function in Witten's formulation of string field theory, Phys.Lett. 179B (1986) 57.

[40] N. Ohta, "Covariant Interacting String Field Theory In The Fock Space Representation," Phys. Rev. D 34 (1986) 3785 [Erratum-ibid. D 35 (1987) 2627].

[41] S. Samuel, The Ghost Vertex In E. Witten's String Field Theory, Phys. Lett. B 181 (1986) 255.

[42] C. Maccaferri and D. Mamone, Star democracy in open string field theory, JHEP 0309 (2003) 049 [arXiv:hep-th/0306252].

[43] A. Kling and S. Uhlmann, "String field theory vertices for fermions of integral weight," JHEP 0307 (2003) 061 [arXiv:hep-th/0306254].

[44] D.Gaiotto, L.Rastelli, A.Sen and B.Zwiebach, Ghost Structure and Closed Strings in Vacuum String Field Theory, [hep-th/0111129].

[45] L.Rastelli, A.Sen and B.Zwiebach, Classical solutions in string field theory around the tachyon vacuum, Adv. Theor. Math. Phys. 5 (2002) 393 [arXiv:hep-th/0102112]. 
[46] L.Rastelli, A.Sen and B.Zwiebach, Star Algebra Spectroscopy, JHEP 0203 (2002) 029 [hep-th/0111281].

[47] D. M. Belov and C. Lovelace, Star products made easy, Phys. Rev. D 68 (2003) 066003 [arXiv:hep-th/0304158].

[48] A.Leclair, M.E.Peskin, C.R.Preitschopf, String Field Theory on the Conformal Plane. (I) Kinematical Principles, Nucl.Phys. B317 (1989) 411.

[49] A. LeClair, M. E. Peskin and C. R. Preitschopf, "String Field Theory on the Conformal Plane. 2. Generalized Gluing," Nucl. Phys. B 317 (1989) 464.

[50] T. Erler and D. J. Gross, "Locality, causality, and an initial value formulation for open string field theory," arXiv:hep-th/0406199.

[51] K.Okuyama, Ghost Kinetic Operator of Vacuum String Field Theory, JHEP 0201 (2002) 027 [hep-th/0201015].

[52] L. Bonora, C. Maccaferri, D. Mamone and M. Salizzoni, "Topics in string field theory," arXiv:hep-th/0304270.

[53] V.A.Kostelecky and R.Potting, Analytical construction of a nonperturbative vacuum for the open bosonic string, Phys. Rev. D 63 (2001) 046007 [hep-th/0008252].

[54] L. Bonora and A. S. Sorin, "Integrable structures in string field theory," Phys. Lett. B 553 (2003) 317 [arXiv:hep-th/0211283].

[55] A. Boyarsky and O. Ruchayskiy, "Integrability in SFT and new representation of KP tau-function," JHEP 0303 (2003) 027 [arXiv:hep-th/0211010].

[56] E. Fuchs and M. Kroyter, "On surface states and star-subalgebras in string field theory," JHEP 0410 (2004) 004 [arXiv:hep-th/0409020].

[57] E. Fuchs and M. Kroyter, "Universal regularization for string field theory," JHEP 0702 (2007) 038 [arXiv:hep-th/0610298].

[58] I. Y. Aref'eva, R. Gorbachev, P. B. Medvedev and D. V. Rychkov, "Descent relations and oscillator level truncation method," Theor. Math. Phys. 150 (2007) 2 [arXiv:hep-th/0606070].

[59] E. Fuchs and M. Kroyter, "Normalization anomalies in level truncation calculations," JHEP 0512 (2005) 031 [arXiv:hep-th/0508010].

[60] C. Maccaferri, Work in progress

[61] see for example: I. B. Frenkel, H. Garland and G. J. Zuckerman, "Semiinfinite Cohomology And String Theory," Proc. Nat. Acad. Sci. 83 (1986) 8442. 